JOURNAL OF THE

AMERICAN MATHEMATICAL SOCIETY

Volume 11, Number 3, July 1998, Pages 521-550

S 0894-0347(98)00269-0

\title{
DECOMPOSING BOREL SETS AND FUNCTIONS AND THE STRUCTURE OF BAIRE CLASS 1 FUNCTIONS
}

\author{
SŁAWOMIR SOLECKI
}

\section{Introduction}

All spaces considered are metric separable and are denoted usually by the letters $X, Y$, or $Z$. $\omega$ stands for the set of all natural numbers. If a metric separable space is additionally complete, we call it Polish; if it is a continuous image of $\omega^{\omega}$ or, equivalently, of a Polish space, it is called Souslin.

The main subject of the present paper is the structure of Baire class 1 functions. Recent developments in Banach space theory, in particular discoveries of Rosenthal, and Bourgain, Fremlin, and Talagrand (see $[R]$ for a survey of these developments), stimulated investigations into the structure of Baire class 1 functions. The interested reader may consult papers by Haydon, Odel, Rosenthal [HOR], Kechris, Louveau [KL], Rosenthal [R2], and references quoted there. (These investigations have already provided new applications in Banach space theory; see, for example, [R1].) In our study of Baire class 1 functions, we will be interested in two decomposition properties, one of them defined by Lusin, the other one by Jayne and Rogers.

First, however, we want to consider a more general problem of determining how difficult it is to represent a Borel set as a union of simpler Borel sets or the graph of a Borel function as a union of the graphs of simpler Borel functions. Using Effective Descriptive Set Theory, in particular Louveau's theorem, we show that if $A \subset X$ is Borel, $X$ Polish, then $A \in \boldsymbol{\Sigma}_{\alpha}^{0}$ or there is a continuous injection $\phi: \omega^{\omega} \rightarrow A$ such that $\phi^{-1}(B)$ is meager for any $B \subset A$ which is $\boldsymbol{\Sigma}_{\alpha}^{0}$. This gives a new proof of J. Stern's result that if a Borel set $A$ is the union of $<\operatorname{cov}(\mathcal{M})$ sets in $\boldsymbol{\Sigma}_{\alpha}^{0}$, then $A$ is itself $\Sigma_{\alpha}^{0} \cdot(\operatorname{cov}(\mathcal{M})$ is the smallest cardinality of a family of meager sets covering $\mathbb{R}$.) We prove similar results for functions. Put, for $f: X \rightarrow Y$ and a family of functions $\mathcal{G}$,

$$
\operatorname{dec}(f, \mathcal{G})=\min \{|\mathcal{F}|: \bigcup \mathcal{F}=X, \forall Z \in \mathcal{F} f \mid Z \in \mathcal{G}\} .
$$

We study these coefficients for various $\mathcal{G}$, in particular for $\mathcal{G}=$ Baire class $\alpha$ functions. We show, for example, that given $f: X \rightarrow Y$ Borel, $X$ Polish, and $\alpha<\omega_{1}$, either $\operatorname{dec}(f$, Baire class $\alpha) \leq \omega$ or there is a continuous injection $\phi: \omega^{\omega} \rightarrow X$ such that $\phi^{-1}(A)$ is meager for any $A \subset X$ with $f \mid A$ in Baire class $\alpha$; thus, in the latter case, $\operatorname{dec}(f$, Baire class $\alpha) \geq \operatorname{cov}(\mathcal{M})$. These results imply that the decomposition coefficients defined in [CMPS] and proved there to be $>\omega$ are actually $\geq \operatorname{cov}(\mathcal{M})$.

Received by the editors May 1, 1997.

1991 Mathematics Subject Classification. Primary 03A15, 26A21, 28A12.

Key words and phrases. Baire class 1 functions, Borel sets, semicontinuous functions, Borel measures, covering of the meager ideal, decomposition of functions. 
In the second part, we apply some of the ideas of the first part to study Baire class 1 functions. We prove two dichotomy results of the following form: a Baire class 1 function "decomposes" into countably many continuous functions or "contains" a very complicated function. Two kinds of decompositions will be considered: decomposition into continuous functions with closed domains (considered first by Jayne and Rogers [JR]) and into continuous functions with arbitrary domains (first considered by Lusin); thus, a function $f: X \rightarrow Y$ will be regarded as simple in the first sense if $X=\bigcup_{n} X_{n}, n \in \omega$, each $X_{n}$ is closed and $f \mid X_{n}$ is continuous, and it will be simple in the latter sense if $X=\bigcup_{n} X_{n}, n \in \omega$, and $f \mid X_{n}$ is continuous for each $n$. To define containment between functions, put for $g: X_{1} \rightarrow Y_{1}$ and $f: X_{2} \rightarrow Y_{2}$

$g \sqsubseteq f$ iff there exist embeddings $\phi: X_{1} \rightarrow X_{2}, \psi: g\left[X_{1}\right] \rightarrow Y_{2}$ with $\psi \circ g=f \circ \phi$.

Now, we identify the functions which will be contained in each complicated, with respect to a decomposition, Baire class 1 function. For the decomposition into continuous functions with closed domains the functions are modeled on the wellknown Lebesgue's example of an increasing function on $[0,1]$ which is continuous exactly at all irrational points; for the decomposition into continuous functions with arbitrary domains the function is the so-called Pawlikowski's function defined in [CMPS]. Here are the precise definitions.

Definition of Lebesgue's functions $L$ and $L_{1}$. Let $Q$ be the set of all points in $2^{\omega}$ which are eventually equal to 1 . For each $x \in Q$ fix a number $a_{x}>0$ so that

1) if $x, y \in Q, x \neq y$, then $a_{x} \neq a_{y}$;

2) $a_{x}<1 / 3^{n_{0}}$, where $n_{0}$ is the smallest natural number such that $x(n)=1$ for $n \geq n_{0}$.

Let $H: 2^{\omega} \rightarrow[0,1]$ be the well-known embedding $H(x)=\sum_{n=0}^{\infty} x(n) / 3^{n+1}$. Let $L, L_{1}: 2^{\omega} \rightarrow \mathbb{R}$ be defined by

$$
L(x)= \begin{cases}H(x), & \text { if } x \notin Q ; \\ H(x)+a_{x}, & \text { if } x \in Q ;\end{cases}
$$

and

$$
L_{1}(x)= \begin{cases}0, & \text { if } x \notin Q \\ a_{x}, & \text { if } x \in Q\end{cases}
$$

Definition of Pawlikowski's function $P$. Let $\omega+1$ have the natural, order topology. Let $P:(\omega+1)^{\omega} \rightarrow \omega^{\omega}$ be defined by $P(\eta)=\gamma, \eta \in(\omega+1)^{\omega}$, where for $n \in \omega$

$$
\gamma(n)= \begin{cases}0, & \text { if } \eta(n)=\omega ; \\ \eta(n)+1, & \text { if } \eta(n)<\omega .\end{cases}
$$

Finally, we can formulate the results. ${ }^{1}$

Let $f: X \rightarrow Y$ be Baire class 1, $X$ Souslin. Then either $X=\bigcup_{n} X_{n}, n \in \omega$, $X_{n}$ closed and $f \mid X_{n}$ continuous, or $L \sqsubseteq f$ or $L_{1} \sqsubseteq f$.

Also, either $X=\bigcup_{n} X_{n}, n \in \omega$, and $f \mid X_{n}$ continuous, or $P \sqsubseteq f$.

\footnotetext{
${ }^{1}$ The following obvious fact is similar to the dichotomy results we intend to prove. Let $d$ : $\omega+1 \rightarrow \omega$ be given by $d(\omega)=0$ and $d(n)=n+1, n \in \omega$, and let $d_{1}: \omega+1 \rightarrow\{0,1\}$ be given by $d_{1}(\omega)=0$ and $d_{1}(n)=1, n \in \omega$. Let $f: X \rightarrow Y$. Then either $f$ is continuous, or $d \sqsubseteq f$ or $d_{1} \sqsubseteq f$.
} 
The first part of the above sentence sharpens a result of Jayne and Rogers from [JR]. The seconed part is related to an old question of Lusin; see remarks below. An interesting feature of the second part is that its proof uses Effective Descriptive Set Theory even though its statement mentions only functions on the first level of Baire hierarchy.

Further, it turns out that $L, L_{1}$, and $P$ are as complicated as any other Baire class 1 function with respect to the decomposition into continuous functions with closed domains, in the case of $L$ and $L_{1}$, and with arbitrary domains, in the case of $P$; thus, the above dichotomy results are in a sense best possible. To phrase it more precisely, put

$$
\operatorname{dec}_{c}(f)=\min \{|\mathcal{F}|: \bigcup \mathcal{F}=X, \forall Z \in \mathcal{F} Z \text { is closed and } f \mid Z \text { is continuous }\}
$$

and

$$
\operatorname{dec}(f)=\min \{|\mathcal{F}|: \bigcup \mathcal{F}=X, \forall Z \in \mathcal{F} f \mid Z \text { is continuous }\},
$$

i.e., $\operatorname{dec}(f)=\operatorname{dec}(f$, continuous $)$. Note that if $g \sqsubseteq f$, then clearly $\operatorname{dec}_{c}(g) \leq \operatorname{dec}_{c}(f)$ and $\operatorname{dec}(g) \leq \operatorname{dec}(f)$. By a result of Cichon and Morayne $[\mathrm{CM}]$,

$$
\sup \left\{\operatorname{dec}_{c}(f): f: X \rightarrow Y, X \text { Souslin, } f \text { Baire class } 1\right\} \leq \mathfrak{d},
$$

where $\mathfrak{d}$ is the smallest cardinality of a dominating subset of $\omega^{\omega}$. We prove that $\operatorname{dec}_{c}(L)=\operatorname{dec}_{c}\left(L_{1}\right)=\mathfrak{d}$. Thus indeed $L$ and $L_{1}$ are as complicated as any other Baire class 1 function as far as decomposing into continuous functions with closed domains is concerned, i.e., $\operatorname{dec}_{c}(L)=\operatorname{dec}_{c}\left(L_{1}\right) \geq \operatorname{dec}_{c}(f)$ for any Baire class 1 function $f$. We prove an analogous result for $P$. Put

$$
\operatorname{dec}=\sup \{\operatorname{dec}(f): f: X \rightarrow Y, X \text { Souslin, } f \text { Baire class } 1\} .
$$

We show that

$$
\operatorname{dec}(P)=\text { dec. }
$$

(This answers two questions of Steprāns [St, Q.7.1 and Q.7.2].) Thus combining the above results, we get that

for any $f: X \rightarrow Y$ Baire class 1, $X$ Souslin, we have $\operatorname{dec}_{c}(f) \leq \omega$ or $\operatorname{dec}_{c}(f)=$ $\mathfrak{d}$, and $\operatorname{dec}(f) \leq \omega$ or $\operatorname{dec}(f)=$ dec.

The alternative $\operatorname{dec}(f) \leq \omega$ or $\operatorname{dec}(f)=\mathbf{d e c}$ can be viewed as a completion, for Baire class 1 functions, of the answer to an old question of Lusin who asked if each Borel function can be decompsed into countably many continuous functions. This was answered in the negative by Keldiš $[\mathrm{K}]$, and an example of a Baire class 1 function which is not decomposable into countably many continuous functions was later found by Adyan and Novikov [AN]. However, the dichotomy $\operatorname{dec}(f) \leq \omega$ or $\operatorname{dec}(f)=\operatorname{dec}$ along with $\operatorname{dec} \geq \operatorname{cov}(\mathcal{M})>\omega$ (see [CMPS]) seem to reflect the situation for Baire class 1 functions much more fully.

The equality $\operatorname{dec}(P)=\mathbf{d e c}$ also gives, via the work of Steprāns, an interesting characterization of dec as the covering coefficient of a certain combinatorially defined $\sigma$-ideal on $\omega^{\omega}$. (It is known that $\operatorname{cov}(\mathcal{M}) \leq \mathbf{d e c} \leq \mathfrak{d}$ [CMPS], and that it is consistent that $\operatorname{cov}(\mathcal{M})<\mathbf{d e c}$, Steprāns [St], and $\mathbf{d e c}<\mathfrak{d}$, Shelah-Steprāns [SS].)

In order to prove $\operatorname{dec}(P)=\mathbf{d e c}$, we define and study complete semicontinuous functions. A lower semicontinuous (lsc) function $F: X \rightarrow[0,1]$ is called $l s c$ complete if each lsc function $f: 2^{\omega} \rightarrow[0,1]$ can be obtained as $F \circ \phi$ for some continuous $\phi: 2^{\omega} \rightarrow X$. We define upper semicontinuous (usc) complete functions 
similarly. Using a Wadge-type game, we give an internal characterization of lsc complete functions as those lsc functions $F: X \rightarrow[0,1]$ for which there is a $\Pi_{2}^{0}$ set $D \subset X$ such that $0 \in F[D]$ and for any open set $U, F[U \cap D]$ is of the form $\left\{y \in[0,1]: y \geq y_{0}\right\}$ or $\left\{y \in[0,1]: y>y_{0}\right\}$ for some $y_{0} \in[0,1]$. Also, we prove the existence of "minimal" lsc complete functions. We give a new proof of the inequality $\operatorname{dec} \geq \operatorname{cov}(\mathcal{M})$, first established in [CMPS], by showing that $\operatorname{dec}(f) \geq \operatorname{cov}(\mathcal{M})$ for any lsc complete $f$. We also have a result that relates the value of $\operatorname{dec}(f)$ to the value of the oscillation ordinal rank $\beta(f)$ studied by Kechris and Louveau in [KL].

If $X$ is a compact, metric space, let $K(X)$ denote the space of all closed subsets of $X$ with the Hausdorff metric. Particular attention has been devoted to the fact that the restriction of the Lebesgue measure to $K([0,1])$ provides a natural example of a complicated usc function [JM], [vMP]. We apply some of the results mentioned above to Borel measures on compact metric spaces $X$ viewed as usc functions on $K(X)$. Using the characterization of complete usc functions, we show that any Borel, probability, nonatomic measure on a compact metric space is usc complete. In fact, we prove a more general version of this result for capacities. This generalizes van Mill and Pol's result for the Lebesgue measure [vMP]. Also, we use the theorem that $\operatorname{dec}=\operatorname{dec}(P)$ to characterize probability, Borel measures $\mu$ on a compact metric space $X$ for which $\operatorname{dec}(\mu)=$ dec, e.g., if $X$ does not have isolated points, then $\operatorname{dec}(\mu)=\mathbf{d e c}$ unless $\mu$ is a finite, convex combination of Dirac measures. This generalizes the result of Jackson and Mauldin that $\operatorname{dec}(\lambda)>\omega$, where $\lambda$ is the Lebesgue measure [JM].

\section{Decomposing Borel sets And Functions INTO SIMPLER BOREL SETS AND FUNCTIONS}

We say that a function $f: X \rightarrow Y$ is in $\mathbf{B}_{\alpha}$ if, for any $U \subset Y$ open, $f^{-1}(U)$ is $\boldsymbol{\Sigma}_{\alpha}^{0}$ in $X$. In particular, $\mathbf{B}_{1}$ is the class of continuous functions. (Note that the enumeration of the $\mathbf{B}_{\alpha}$ 's, as that of the $\boldsymbol{\Sigma}_{\alpha}^{0}$ 's, starts with $\alpha=1$.) Also define $f$ : $X \rightarrow \mathbb{R}$ to be in $\mathbf{L}_{\alpha}\left(\mathbf{U}_{\alpha}\right.$, respectively) if $f^{-1}((r, \infty))\left(f^{-1}((-\infty, r))\right.$, respectively) is $\boldsymbol{\Sigma}_{\alpha}^{0}$ in $X$ for all $r \in \mathbb{R}$. Thus $\mathbf{L}_{1}$ and $\mathbf{U}_{1}$ are the classes of lower and upper semicontinuous functions, respectively. ${ }^{2}$ Note that for real functions for each $1 \leq$ $\alpha<\omega_{1}$, we have $\mathbf{B}_{\alpha} \subset \mathbf{U}_{\alpha} \cap \mathbf{L}_{\alpha}$ and $\mathbf{U}_{\alpha} \cup \mathbf{L}_{\alpha} \subset \mathbf{B}_{\alpha+1}$. The classes $\mathbf{B}_{\alpha}$ are closely related to Baire classes of Borel functions, in fact, all Baire classes occur among the $\mathbf{B}_{\alpha}$ 's. Recall that $f: X \rightarrow Y$ is Baire class 1 , or is in $\mathcal{B}_{1}$, if preimages of open sets via $f$ are $\boldsymbol{\Sigma}_{2}^{0}$. A function $f: X \rightarrow Y$ is Baire class $\alpha$, or is in $\mathcal{B}_{\alpha}$, if it is the pointwise limit of a sequence of functions in $\bigcup_{\gamma<\alpha} \mathcal{B}_{\gamma}$. The classical theorem of Lebesgue, Hausdorff, and Banach states that $\mathcal{B}_{\alpha}=\mathbf{B}_{\alpha+1}$ for $1 \leq \alpha<\omega_{1}$. In particular, Baire class 1 functions constitute $\mathbf{B}_{2}$. Let $\operatorname{cov}(\mathcal{M})$ be the smallest cardinality of a family of meager sets covering $\mathbb{R}$. Recall that the Gandy-Harrington topology on a recursively presented Polish space is the topology generated by all $\Sigma_{1}^{1}$ sets and that it is strong Choquet. (See [HKL] for some background on the Gandy-Harrington topology.)

We say that a set $D$ separates $A$ and $B$ if $A \subset D$ and $D \cap B=\emptyset$. We will use the following theorem due to Louveau (see [L1]):

\footnotetext{
${ }^{2}$ The enumeration of the first $\omega$ classes $\mathbf{B}_{\alpha}, \mathbf{L}_{\alpha}$, and $\mathbf{U}_{\alpha}$ here differs from that in [CMPS] by 1 .
} 
Let $A_{0}, A_{1}$ be $\Sigma_{1}^{1}$ sets such that for some $D \in \Pi_{\alpha}^{0}, 1 \leq \alpha<\omega_{1}^{C K}, A_{0} \subset D$ and $A_{1} \cap D=\emptyset$ modulo sets meager in the Gandy-Harrington topology. Then $A_{0}$ and $A_{1}$ can be separated by a set from $\Pi_{\alpha}^{0}\left(\Delta_{1}^{1}\right)$.

Let $\mathcal{A}$ be a family of subsets of a Polish space $X$. Let $C \subset X$, and let $1 \leq \alpha<\omega_{1}$. We say that

- $\mathcal{A}$ is $\Pi_{\alpha}^{0}$ on $C$ iff $\forall A \in \mathcal{A} \exists D \in \Pi_{\alpha}^{0} A \cap C \subset D \subset A$;

- $\mathcal{A}$ is relatively $\Pi_{\alpha}^{0}$ on $C$ iff $\forall A \in \mathcal{A} \exists D \in \Pi_{\alpha}^{0} A \cap C \subset D \cap C \subset A$ (i.e., $A \cap C=D \cap C)$.

Lemma 2.1. Let $\mathcal{A}_{1}, \mathcal{A}_{2}$ be countable families of Borel subsets of a Polish space $X$, and let $1<\beta<\omega_{1}$. Then precisely one of the following two possibilities holds:

(i) $X=\bigcup_{n} C_{n}$ and, for each $n \in \omega, \mathcal{A}_{1}$ or $\mathcal{A}_{2}$ is $\Pi_{\alpha}^{0}$ (relatively $\Pi_{\alpha}^{0}$, respectively) on $C_{n}$ for some $\alpha<\beta$.

(ii) There is a continuous injection $\phi: \omega^{\omega} \rightarrow \bigcup \mathcal{A}_{1} \cap \bigcup \mathcal{A}_{2}$ such that if $\mathcal{A}_{1}$ or $\mathcal{A}_{2}$ is $\boldsymbol{\Pi}_{\alpha}^{0}$ (relatively $\boldsymbol{\Pi}_{\alpha}^{0}$, respectively) on $C$ for some $\alpha<\beta$, then $\phi^{-1}(C)$ is meager.

Proof. We will prove the statements for " $\boldsymbol{\Pi}_{\alpha}^{0}$ on $C$ " and "relatively $\boldsymbol{\Pi}_{\alpha}^{0}$ on $C$ " simultaneously. Let $\mathcal{A}$ be a countable family of Borel subsets of $X$. Fix $A \subset X \times \omega$ such that

$$
\mathcal{A}=\{\{x \in X:(x, n) \in A\}: n \in \omega\} .
$$

Since the argument below relativizes, we can assume that $X$ is a recursively presented Polish space, $\beta<\omega_{1}^{C K}$, and $A \in \Delta_{1}^{1}$. Note that for $C \subset X$

$\mathcal{A}$ is $\Pi_{\alpha}^{0}$ on $C$ iff $(C \times \omega) \cap A$ and $(X \times \omega) \backslash A$ can be separated by a $\Pi_{\alpha}^{0}$ set, and also

$\mathcal{A}$ is relatively $\Pi_{\alpha}^{0}$ on $C$ iff

$$
(C \times \omega) \cap A \text { and }(C \times \omega) \backslash A \text { can be separated by a } \Pi_{\alpha}^{0} \text { set. }
$$

Let $\Phi: \mathcal{P}(X) \rightarrow \mathcal{P}(X)$ denote either the identity function, or the constant function $\Phi(C)=X$ for all $C \in \mathcal{P}(X)$. Put

$$
\begin{aligned}
& P^{\Phi}=\left\{C \subset X: C \in \Sigma_{1}^{1}\right. \text { and } \\
& \left.\quad(C \times \omega) \cap A \text { and }(\Phi(C) \times \omega) \backslash A \text { can be separated by a set in } \bigcup_{\alpha<\beta} \Pi_{\alpha}^{0}\right\} .
\end{aligned}
$$

Claim 1. $\cup P^{\Phi}$ is $\Pi_{1}^{1}$.

If $C \in P^{\Phi}$, then, by Louveau's theorem, there is a $D \in \Pi_{\alpha}^{0}\left(\Delta_{1}^{1}\right)$, for some $\alpha<\beta$, which separates the $\Sigma_{1}^{1}$ sets $(C \times \omega) \cap A$ and $(\Phi(C) \times \omega) \backslash A$. Put

$$
C^{\prime}=\{x \in X: \forall n(x, n) \in(D \cap A) \cup((X \times \omega) \backslash(D \cup A))\} .
$$

Then $C \subset C^{\prime}, C^{\prime} \in \Sigma_{1}^{1}$, and, as is easy to see, $D$ separates $\left(C^{\prime} \times \omega\right) \cap A$ and $\left(\Phi\left(C^{\prime}\right) \times \omega\right) \backslash A$, i.e., $C^{\prime} \in P^{\Phi}$. Thus

$$
\bigcup P^{\Phi}=\left\{x \in X: \exists D \in \bigcup_{\alpha<\beta} \Pi_{\alpha}^{0}\left(\Delta_{1}^{1}\right) \forall n(x, n) \in(D \cap A) \cup((X \times \omega) \backslash(D \cup A))\right\},
$$

which is $\Pi_{1}^{1}$.

Below in this proof all topological notions - meager, $G_{\delta}$, etc. - refer to the GandyHarrington topology. 
Claim 2. Let $C \subset X$ be such that $(C \times \omega) \cap A$ and $(\Phi(C) \times \omega) \backslash A$ can be separated by a set from $\bigcup_{\alpha<\beta} \Pi_{\alpha}^{0}$. Then there are $C_{n} \in P^{\Phi}, n \in \omega$, such that $C \backslash \bigcup_{n} C_{n}$ is meager.

There exist $C_{n} \in \Sigma_{1}^{1}, n \in \omega$, such that $C_{n} \backslash C$ does not contain a nonmeager set with the Baire property, for each $n$, and $C \backslash \bigcup_{n} C_{n}$ is meager. Let $D \in \bigcup_{\alpha<\beta} \Pi_{\alpha}^{0}$ separate $(C \times \omega) \cap A$ and $(\Phi(C) \times \omega) \backslash A$. Note that $\left(C_{n} \times \omega\right) \cap A \subset D$ and $\left(\left(\Phi\left(C_{n}\right) \times \omega\right) \backslash A\right) \cap D=\emptyset$ modulo meager sets. Thus, by Louveau's theorem, for each $n \in \omega$ there is a set in $\bigcup_{\alpha<\beta} \Pi_{\alpha}^{0}\left(\Delta_{1}^{1}\right)$ which separates $\left(C_{n} \times \omega\right) \cap A$ and $\left(\Phi\left(C_{n}\right) \times \omega\right) \backslash A$. Therefore $C_{n} \in P^{\Phi}$.

Let $A_{1}, A_{2}$ and $P_{1}^{\Phi}, P_{2}^{\Phi}$ be defined as in (1) and (2) for $\mathcal{A}=\mathcal{A}_{1}$ and $\mathcal{A}=\mathcal{A}_{2}$, respectively. If $\bigcup P_{1}^{\Phi} \cup \bigcup P_{2}^{\Phi} \supset \bigcup \mathcal{A}_{1} \cap \bigcup \mathcal{A}_{2}$, then actually $\bigcup P_{1}^{\Phi} \cup \bigcup P_{2}^{\Phi}=X$, as $X \backslash \cup \mathcal{A}_{i}=X \backslash\left\{x \in X: \exists n(x, n) \in A_{i}\right\} \in P_{i}^{\Phi}, i=1,2$, whence (i) holds. If not, put

$$
E_{1}=\bigcup \mathcal{A}_{1} \cap \bigcup \mathcal{A}_{2} \backslash \bigcup P_{1}^{\Phi} \cup \bigcup P_{2}^{\Phi} .
$$

By Claim $1, E_{1}$ is a nonempty $\Sigma_{1}^{1}$ set. If $C \subset X$ is such that $(C \times \omega) \cap A_{i}$ and $(\Phi(C) \times \omega) \backslash A_{i}$ can be separated by a $\Pi_{\alpha}^{0}$ set, for some $\alpha<\beta$, then, by Claim $2, C \cap E_{1}$ is meager. Note that $(\{x\} \times \omega) \cap A_{i}$ and $(\Phi(\{x\}) \times \omega) \backslash A_{i}$ can be separated by a $\Pi_{1}^{0}$ set for any $x \in X$; thus $E_{1}$ does not have isolated points. Let $\left\{B_{n}: n \in \omega\right\}$ be a countable basis of $E_{1}$. Put $E_{2}=E_{1} \backslash \bigcup_{n}\left(\bar{B}_{n} \backslash B_{n}\right)$. Then $E_{2}$ is a dense $G_{\delta}$ in $E_{1}$, whence it is strong Choquet (see [HKL, Proposition 2.1(iii)]). Since it is clearly regular and has countable basis, it is Polish by Choquet's theorem. Moreover, since $E_{2}$ does not have isolated points, we can find a dense $G_{\delta}$ subset of $E_{2}$ homeomorphic to $\omega^{\omega}$. This finishes the proof of the lemma.

Theorem 2.2. Let $X$ be a Polish space, and let $1<\beta<\omega_{1}$. Let $A \subset X$ be Borel. Then either $A \in \boldsymbol{\Sigma}_{\beta}^{0}$, or there is a continuous injection $\phi: \omega^{\omega} \rightarrow A$ such that for any $C \subset A, C \in \mathbf{\Sigma}_{\beta}^{0}, \phi^{-1}(C)$ is meager in $\omega^{\omega}$.

Proof. Let $\mathcal{A}_{1}=\mathcal{A}_{2}=\{A\}$. If $X=\bigcup_{n} C_{n}$ and, for each $n \in \omega, \mathcal{A}_{1}$ is $\Pi_{\alpha}^{0}$ on $C_{n}$ for some $\alpha<\beta$, then $A$ is $\boldsymbol{\Sigma}_{\beta}^{0}$. Otherwise, from Lemma 2.1, we get a continuous injection $\phi$ as required.

I was informed by A. Miller that the following corollary was proved by Stern [Sr, Theorem 3.2]. Stern's proof is different from the one presented here and uses Steel's forcing. Also, [BD, Theorem 2] contains a similar but weaker result. The corollary immediately follows from Theorem 2.2 if $\alpha>1$ and is trivial if $\alpha=1$.

Corollary 2.3. Let $A$ be a Borel set in a Polish space, and let $1 \leq \beta<\omega_{1}$. Assume $A$ is the union of $<\operatorname{cov}(\mathcal{M})$ sets in $\boldsymbol{\Sigma}_{\beta}^{0}$. Then $A \in \boldsymbol{\Sigma}_{\beta}^{0}$.

Theorem 2.4. Let $X$ be a Polish space, and let $1<\beta<\omega_{1}$. Let $\mathcal{G}$ be one of the following:

$$
\bigcup_{\alpha<\beta} \mathbf{B}_{\alpha}, \bigcup_{\alpha<\beta} \mathbf{L}_{\alpha}, \bigcup_{\alpha<\beta} \mathbf{U}_{\alpha}, \bigcup_{\alpha<\beta} \mathbf{L}_{\alpha} \cup \mathbf{U}_{\alpha} .
$$

Let $f: X \rightarrow Y$ be a Borel function with $Y=\mathbb{R}$ if we consider the last three classes. Then either $\operatorname{dec}(f, \mathcal{G}) \leq \omega$, or there is a continuous injection $\phi: \omega^{\omega} \rightarrow X$ such that if $f \mid C \in \mathcal{G}$, then $\phi^{-1}(C)$ is meager, so $\operatorname{dec}(f, \mathcal{G}) \geq \operatorname{cov}(\mathcal{M})$. 
Proof. Put $\mathcal{A}_{1}=\mathcal{A}_{2}=\left\{f^{-1}\left(Y \backslash V_{n}\right): n \in \omega\right\}$, where $\left\{V_{n}: n \in \omega\right\}$ is a countable topological basis of $Y$. Note, that $\mathcal{A}_{1}$ is relatively $\Pi_{\alpha}^{0}$ on $C \subset X$ iff $f \mid C \in \mathbf{B}_{\alpha}$. Thus, an application of Lemma 2.1 similar to the one in Theorem 2.2 gives the conclusion for $\mathcal{G}=\bigcup_{\alpha<\beta} \mathbf{B}_{\alpha}$. To obtain it for $\mathcal{G}=\bigcup_{\alpha<\beta} \mathbf{L}_{\alpha}, \mathcal{G}=\bigcup_{\alpha<\beta} \mathbf{U}_{\alpha}$, and $\mathcal{G}=\bigcup_{\alpha<\beta} \mathbf{L}_{\alpha} \cup \mathbf{U}_{\alpha}$ apply a similar argument respectively to the families $\mathcal{A}_{1}=\mathcal{A}_{2}=\left\{f^{-1}((-\infty, q]): q \in \mathbb{Q}\right\}, \mathcal{A}_{1}=\mathcal{A}_{2}=\left\{f^{-1}([q, \infty)): q \in \mathbb{Q}\right\}$, and $\mathcal{A}_{1}=\left\{f^{-1}((-\infty, q]): q \in \mathbb{Q}\right\}, \mathcal{A}_{2}=\left\{f^{-1}([q, \infty)): q \in \mathbb{Q}\right\}$.

Remark. By the remarks at the beginning of this section it follows from the above theorem that its conculsion holds for $\mathcal{G}=\mathcal{B}_{\alpha}$ (= Baire class $\alpha$ functions).

It was proved in [CMPS, Corollary 3.3] that there is an $f \in \mathbf{B}_{\beta}$ with

$$
\operatorname{dec}\left(f, \bigcup_{\alpha<\beta} \mathbf{L}_{\alpha} \cup \mathbf{U}_{\alpha}\right)>\omega,
$$

and also [CMPS, Theorem 5.7] that there is an $f \in \mathbf{B}_{2}$, that is $f$ Baire class 1, with

$$
\operatorname{dec}\left(f, \mathbf{L}_{1} \cup \mathbf{U}_{1}\right) \geq \operatorname{cov}(\mathcal{M}) .
$$

Laczkovich showed that for any $1 \leq \beta<\omega_{1}$ there is an $f \in \mathbf{L}_{\beta}$ with $\operatorname{dec}\left(f, \mathbf{B}_{\beta}\right)>\omega$ (see $[\mathrm{CM}]$ for a proof); and by [CMPS, Theorem 5.5] there is an $f \in \mathbf{L}_{1}$ that is $f$ lower semicontinous, with $\operatorname{dec}\left(f, \mathbf{B}_{1}\right) \geq \operatorname{cov}(\mathcal{M})$. The next corollary improves on these results. Let me first mention, however, that Steprāns established in [St] the consistency with ZFC of the existence of $f \in \mathbf{L}_{1}$ such that $\operatorname{dec}\left(f, \mathbf{B}_{1}\right)>\operatorname{cov}(\mathcal{M})$.

Corollary 2.5. Let $X$ be Polish uncountable.

(i) For each $1<\beta<\omega_{1}$ there exists $f: X \rightarrow \mathbb{R}, f \in \mathbf{B}_{\beta}$, such that

$$
\operatorname{dec}\left(f, \bigcup_{\alpha<\beta} \mathbf{L}_{\alpha} \cup \mathbf{U}_{\alpha}\right) \geq \operatorname{cov}(\mathcal{M}) .
$$

(ii) For each $1 \leq \beta<\omega_{1}$ there exists $f: X \rightarrow \mathbb{R}, f \in \mathbf{L}_{\beta}$, such that

$$
\operatorname{dec}\left(f, \mathbf{B}_{\beta}\right) \geq \operatorname{cov}(\mathcal{M}) .
$$

Proof. By [CMPS, Corollary 3.3], there is $f: X \rightarrow \mathbb{R}, f \in \mathbf{B}_{\beta}$, with

$$
\operatorname{dec}\left(f, \bigcup_{\alpha<\beta} \mathbf{L}_{\alpha} \cup \mathbf{U}_{\alpha}\right)>\omega .
$$

Thus (i) follows from Theorem 2.4. To prove (ii), use the fact that there is an $f: X \rightarrow \mathbb{R}, f \in \mathbf{L}_{\beta}$, such that $\operatorname{dec}\left(f, \mathbf{B}_{\beta}\right)>\omega[\mathrm{CM}$, Corollary 3.4] and apply Theorem 2.4.

Remarks. 1. By the proof of Theorem 4.8 from [CMPS], for $1 \leq \beta<\omega_{1}$ and any $f \in \mathbf{B}_{\beta+1}$ there is a $g \in \mathbf{L}_{\beta}$ such that $\operatorname{dec}\left(g, \mathbf{B}_{\beta}\right) \geq \operatorname{dec}\left(f, \mathbf{L}_{\beta} \cup \mathbf{U}_{\beta}\right)$. Thus (ii) in our Corollary 2.5 actually follows from (i).

2. I do not know whether the method employed here can be used to show that the more subtle decomposition coefficients studied by Morayne in $[\mathrm{M}]$ are also $\geq \operatorname{cov}(\mathcal{M})$. Perhaps the refined version of Louveau's theorem from [L2] can be of some help. 


\section{Decomposing Baire class 1 functions INTO CONTINUOUS FUNCTIONS WITH CLOSED DOMAINS}

Recall that a function $f$ is Baire class 1 if preimages via $f$ of open sets are $\boldsymbol{\Sigma}_{2}^{0}$.

In [JR, Theorem 1], Jayne and Rogers proved that for any function $f: X \rightarrow Y$, $X$ Souslin, either there are closed sets $X_{n} \subset X, n \in \omega$, such that $\bigcup_{n} X_{n}=X$ and $f \mid X_{n}$ is continuous, or there is an $F_{\sigma}$ set $A \subset Y$ such that $f^{-1}(A)$ is not $F_{\sigma}$. The next result - the first dichotomy theorem for Baire class 1 functions - sharpens Jayne and Rogers's theorem. (For a derivation of [JR, Theorem 1] from Theorem 3.1 see the remark following the proof of Theorem 3.1.)

Theorem 3.1. Let $f: X \rightarrow Y$ be Baire class 1, $X$ Souslin. Then precisely one of the following holds:

(i) There are closed sets $X_{n} \subset X, n \in \omega$, such that $\bigcup_{n} X_{n}=X$ and $f \mid X_{n}$ is continuous.

(ii) $L \sqsubseteq f$ or $L_{1} \sqsubseteq f$.

We will need a few auxiliary notions. For a sequence of sets $A_{k} \subset X, k \in \omega$, and $x \in X$, we write $A_{k} \rightarrow x$ if each $A_{k}$ is nonempty and, for any $\epsilon>0, A_{k} \subset B(x, \epsilon)$ for $k$ large enough. A function $f: X \rightarrow Y$ is strongly discontinuous at $x \in X$ if there exist a sequence of open sets $V_{k} \subset X$ and an open set $U \subset Y$ such that $V_{k} \rightarrow x, f(x) \in U$ and $f\left[V_{k}\right] \cap U=\emptyset$. A point $x \in X$ is $f$-isolated if there is an open set $U \subset Y$ such that $f^{-1}(U)=\{x\}$. By $\operatorname{osc}(f, x)$ we denote the oscillation of $f$ at $x$.

First, we give characterizations of $L$ and $L_{1}$.

Lemma 3.2. Let $g: 2^{\omega} \rightarrow Y$. Assume each $x \in Q$ is g-isolated, $g$ is continuous at each $x \in 2^{\omega} \backslash Q$, and, given $\epsilon>0, \operatorname{osc}(g, x)<\epsilon$ for all but finitely many points in $Q$.

(i) If $g \mid\left(2^{\omega} \backslash Q\right)$ is an embedding, then $\psi_{0}: L\left[2^{\omega}\right] \rightarrow Y$ given by $\psi_{0}(L(x))=g(x)$ is a well-defined embedding, and $\psi_{0} \circ L=g$.

(ii) If $g \mid\left(2^{\omega} \backslash Q\right)$ is constant, then $\psi_{1}: L_{1}\left[2^{\omega}\right] \rightarrow Y$ given by $\psi_{1}\left(L_{1}(x)\right)=g(x)$ is a well-defined embedding, and $\psi_{1} \circ L_{1}=g$.

Proof. (i) Since $g \mid\left(2^{\omega} \backslash Q\right)$ is 1-to-1 and each $x \in Q$ is $g$-isolated, $g$ is 1-to-1. Also $L$ is 1-to-1, thus $\psi_{0}$ is well-defined and 1-to-1. Let $L\left(x_{n}\right) \rightarrow L(x)$ and $L\left(x_{n}\right) \neq L(x)$. Clearly $x \in 2^{\omega} \backslash Q$ and $x_{n} \rightarrow x$. Since $x$ is a continuity point of $g, g\left(x_{n}\right) \rightarrow g(x)$. Thus, $\psi_{0}\left(L\left(x_{n}\right)\right) \rightarrow \psi_{0}(L(x))$; whence $\psi_{0}$ is continuous. Assume $g\left(x_{n}\right) \rightarrow g(x)$. Since each $x \in Q$ is $g$-isolated, $x \in 2^{\omega} \backslash Q$. Since $\operatorname{osc}\left(g, x_{n}\right) \rightarrow 0$, we can find $z_{n} \in 2^{\omega} \backslash Q$ such that $d\left(z_{n}, x_{n}\right) \rightarrow 0$ and $d\left(g\left(z_{n}\right), g\left(x_{n}\right)\right) \rightarrow 0$. Thus $g\left(z_{n}\right) \rightarrow g(x)$. Since $g \mid 2^{\omega} \backslash Q$ is an embedding, $z_{n} \rightarrow x$, whence $x_{n} \rightarrow x$. Thus $L\left(x_{n}\right) \rightarrow L(x)$, i.e., $\psi_{0}^{-1}\left(g\left(x_{n}\right)\right) \rightarrow \psi^{-1}(g(x))$; whence $\psi$ is an embedding.

(ii) If $L_{1}(x)=L_{1}(y)$, then $x, y \in 2^{\omega} \backslash Q$ or $x=y$, so $g(x)=g(y)$. Thus, $\psi_{1}$ is welldefined. Note that if $L_{1}(x) \neq L_{1}(y)$, then $x \neq y$ and $x \in Q$ or $y \in Q$. Since each element of $Q$ is $g$-isolated, $g(x) \neq g(y)$. Thus $\psi_{1}$ is 1-to-1. Let $L_{1}\left(x_{n}\right) \rightarrow L_{1}(x)$ and $L_{1}\left(x_{n}\right) \neq L_{1}(x)$. Then clearly $x_{n} \in Q$ and $x \in 2^{\omega} \backslash Q$. Since osc $\left(g, x_{n}\right) \rightarrow 0$, there are $z_{n} \in 2^{\omega} \backslash Q$ with $d\left(g\left(x_{n}\right), g\left(z_{n}\right)\right) \rightarrow 0$. But $g\left(z_{n}\right)=g(x)$. Thus $g\left(x_{n}\right) \rightarrow g(x)$, so $\psi\left(L_{1}\left(x_{n}\right)\right) \rightarrow \psi\left(L_{1}(x)\right)$. So $\psi$ is continuous. Since $L_{1}\left[2^{\omega}\right]$ is compact, $\psi$ is an embedding.

Lemma 3.3. Let $f: X \rightarrow Y$. Assume the sets of all continuity and of all discontinuity points of $f$ are both dense. For $\epsilon>0$, let $S_{\epsilon}$ be the set of all strong 
discontinuity points at which the oscillation of $f$ is $<\epsilon$. Then for any $\emptyset \neq U \subset X$ open $f\left[U \cap S_{\epsilon}\right]$ is infinite.

Proof. Let $S$ be the set of all strong discontinuity points of $f$. Note that if there is a sequence $x_{n} \rightarrow x, x_{n}$ are continuity points of $f$ and $f\left(x_{n}\right) \nrightarrow f(x)$, then $x \in S$. To see this, first find a subsequence $\left(x_{n_{k}}\right)$ of $\left(x_{n}\right)$ and an open set $V \subset Y$ such that $f(x) \in V$ and $f\left(x_{n_{k}}\right) \notin V$. Since each $x_{n_{k}}$ is a continuity point, we can find open sets $W_{k} \ni x_{n_{k}}$ and an open set $V^{\prime} \subset V$ such that $f(x) \in V^{\prime}$ and $f\left[W_{k}\right] \cap V^{\prime}=\emptyset$. By making $W_{k}$ small in diameter, we ensure that $W_{k} \rightarrow x$.

Now, we show that $S$ is dense. Let $\emptyset \neq U \subset X$ be open. Let $x \in U$ be a discontinuity point of $f$. Let $x_{n} \in U$ and $V \subset Y$ open be such that $x_{n} \rightarrow x$, $f(x) \in V$, and $f\left(x_{n}\right) \notin V$. Let $y_{k}^{n}, n, k \in \omega$, be continuity points of $f$ such that $y_{k}^{n} \in U$ and $y_{k}^{n} \rightarrow x_{n}$. If $f\left(y_{k}^{n}\right) \not f\left(x_{n}\right)$ for some $n$, then $x_{n} \in S$. If $f\left(y_{k}^{n}\right) \rightarrow f\left(x_{n}\right)$ for all $n$, then we can choose a "diagonal" sequence $y_{k_{n}}^{n}$ so that $y_{k_{n}}^{n} \rightarrow x$ and $f\left(y_{k_{n}}^{n}\right) \nrightarrow f(x)$, so $x \in S$. In any case, $S \cap U \neq \emptyset$.

Let $\emptyset \neq U \subset X$ be open. We construct by induction a sequence $x_{n} \in S \cap U$ such that $f\left(x_{n}\right) \neq f\left(x_{m}\right)$ if $n \neq m$. Let $x_{0} \in S \cap U$. Since $x_{0} \in S$, there is $\emptyset \neq V_{0} \subset U$ with $f\left(x_{0}\right) \notin f\left[V_{0}\right]$. Let $x_{1} \in S \cap V_{0}$. Find $\emptyset \neq V_{1} \subset V_{0}$ open so that $f\left(x_{1}\right) \notin f\left[V_{1}\right]$. Let $x_{2} \in S \cap V_{1}$. Continuing this procedure, we obtain a sequence $\left(x_{n}\right)$ as required. Thus, for any $\emptyset \neq U \subset X$ open $f[S \cap U]$ is infinite. Since $S_{\epsilon}=S \cap\{x \in X: \operatorname{osc}(f, x)<\epsilon\}$ and $\{x \in X: \operatorname{osc}(f, x)<\epsilon\}$ is dense, as it contains all continuity points of $f$, and obviously open, we also have that $f\left[S_{\epsilon} \cap U\right]$ is infinite.

Lemma 3.4. Let $f: X \rightarrow Y, X$ Polish, be Baire class 1. Assume that the set of all discontinuity points of $f$ is dense. Then there is a compact perfect set $K \subset X$ and a countable set $D \subset K$ such that

(i) $D$ is dense in $K$;

(ii) each $x \in D$ is $f \mid K$-isolated;

(iii) given $\epsilon>0, \operatorname{osc}(f \mid K, x)<\epsilon$ for all but finitely many points in $D$.

Proof. Fix $\phi: \omega \rightarrow \omega$ such that $\phi(n) \leq n$ and $\forall n \exists^{\infty} k n=\phi(k)$. We construct sequences $F_{n} \subset X$ closed and $q_{n} \in X$ so that

1) $F_{n+1} \subset F_{n}$;

2) $\left\{q_{k}: k \leq n\right\} \subset F_{n}$;

3) $\forall x \in F_{n} \exists k \leq n d\left(x, q_{k}\right) \leq 1 /(n+1)$;

4) $d\left(q_{n+1}, q_{\phi(n)}\right) \leq 1 /(n+1)$;

5) $q_{n}$ is $f \mid F_{n}$-isolated;

6) $\operatorname{osc}\left(f \mid F_{n}, q_{n}\right)<1 /(n+1)$;

7) $\operatorname{int}\left(F_{n}\right)$ is dense in $F_{n}$.

We will put $K=\bigcap_{n} F_{n}$ and $D=\left\{q_{n}: n \in \omega\right\}$. $K$ is clearly closed and by 3) totally bounded, whence compact. By 2), $D \subset K$, and by 3) $D$ is dense in $K$. By 4), as $\forall n \exists^{\infty} k n=\phi(k), D$ is dense-in-itself; thus $K$ is perfect. Since $K \subset F_{n}$, each $q_{n}$ is $f \mid K$-isolated by 5$)$, and $\operatorname{osc}\left(f \mid K, q_{n}\right)<1 /(n+1)$ by 6$)$.

Since $f$ is Baire class 1 , continuity points of $f$ are dense in $X$; thus, we can apply Lemma 3.3. Let $q_{0} \in S_{1}$. There is $V \subset Y$ open and a sequence of open sets $V_{k} \subset X$ such that $V_{k} \rightarrow q_{0}, V_{k} \subset B\left(q_{0}, 1\right), f\left(q_{0}\right) \in V$, and $f\left[V_{k}\right] \cap V=\emptyset$. Put $F_{0}=\left\{q_{0}\right\} \cup \bigcup_{k} \bar{V}_{k}$. Assume $F_{n}$ and $q_{k}, k \leq n$, have been defined. Let $\emptyset \neq U \subset B\left(q_{\phi(n)}, 1 /(n+2)\right) \cap F_{n}$ be open. (This is possible by 7$)$.) By Lemma 3.3, find $p_{0}, \ldots, p_{n+1} \in U \cap S_{1 /(n+1)}$ so that $f\left(p_{i}\right) \neq f\left(p_{j}\right)$ if $i \neq j$. Let $W_{i} \subset Y$ be open 
such that $f\left(p_{i}\right) \in W_{i}$ and $\bar{W}_{i} \cap \bar{W}_{j}=\emptyset$ if $i \neq j$. For each $k \leq n$ there is at most one $i \leq n+1$ such that $f^{-1}\left(\bar{W}_{i}\right)$ is comeager in $W \cap F_{n}$ for some open $W \ni q_{k}$. Thus, by the pigeonhole principle, there is $i_{0} \leq n+1$ such that for each $k \leq n$, $X \backslash f^{-1}\left(\bar{W}_{i_{0}}\right)$ is not meager in any neighborhood of $q_{k}$ in $F_{n}$. But $X \backslash f^{-1}\left(\bar{W}_{i_{0}}\right)$ is $F_{\sigma}$, so using 7$)$, we can find $V_{m}^{k} \subset\left(X \backslash f^{-1}\left(\bar{W}_{i_{0}}\right)\right) \cap F_{n}, n \in \omega$, open and such that $V_{m}^{k} \subset B\left(q_{k}, 1 /(n+2)\right), V_{m}^{k} \rightarrow q_{k}$. By the choice of the $p_{i}$ 's and by making $W_{i_{0}}$ smaller if necessary, we can find $V_{m} \subset B\left(p_{i_{0}}, 1 /(n+2)\right) \cap\left(X \backslash f^{-1}\left(W_{i_{0}}\right)\right) \cap F_{n}$ open with $V_{m} \rightarrow p_{i_{0}}$. Put $q_{n+1}=p_{i_{0}}$ and

$$
F_{n+1}=\left\{q_{k}: k \leq n+1\right\} \cup \bigcup_{k \leq n} \bigcup_{m} \overline{V_{m}^{k}} \cup \bigcup_{m} \bar{V}_{m} .
$$

All the requirements 1)-7) are easy to check.

The following lemma is certainly well known. Recall that a set $H \subset \omega^{\omega}$ is called $\sigma$-bounded if there are $x_{n} \in \omega^{\omega}, n \in \omega$, such that for any $x \in H$ there is an $n \in \omega$ with $x(k) \leq x_{n}(k)$ for all $k \in \omega$.

Lemma 3.5. Let $f: \omega^{\omega} \rightarrow Y$ be continuous. Then there is a closed, non- $\sigma$ bounded set $H_{1} \subset \omega^{\omega}$ such that $f \mid H_{1}$ is constant, or there exists a closed, non- $\sigma$ bounded set $H_{2} \subset \omega^{\omega}$ such that $f \mid H_{2}$ is an embedding.

Proof. Case 1. $\exists U \subset \omega^{\omega}$ open, nonempty and such that $f[U]$ is finite.

Then, since $f$ is continuous, there is $\emptyset \neq V \subset U$ open and such that $f \mid V$ is constant. Put $H_{1}=\overline{V^{\prime}}$ for some open nonempty $V^{\prime}$ with $\overline{V^{\prime}} \subset V$.

Case 2. $\forall U \subset \omega^{\omega}$ open, nonempty, $f[U]$ is infinite.

Define recursively $\sigma_{s} \in \omega^{<\omega}, s \in \omega^{<\omega}$, so that

1) if $s \subset t$, then $\sigma_{s} \subset \sigma_{t}$ and if $s$ and $t$ are incompatible, then so are $\sigma_{s}$ and $\sigma_{t}$;

2) $\left\{f\left[N_{\sigma_{s * n}}\right]: n \in \omega\right\}$ is a discrete family;

3) $\operatorname{diam}\left(f\left[N_{\sigma_{s}}\right]\right) \leq 1 /(\operatorname{lh}(s)+1)$;

4) $\left\{\sigma_{s * n}(l): n \in \omega\right\}$ is infinite, where $l=l h\left(\sigma_{s}\right)$.

Assume $\sigma_{s}$ is defined. Let $l=l h\left(\sigma_{s}\right)$. Since $f\left[N_{\sigma_{s} * p}\right]$ is infinite for each $p \in \omega$, we can find a sequence $x_{n} \in N_{\sigma_{s}}, n \in \omega$, such that $x_{n_{1}}(l) \neq x_{n_{2}}(l)$ and $f\left(x_{n_{1}}\right) \neq f\left(x_{n_{2}}\right)$ if $n_{1} \neq n_{2}$. We can assume that $\left\{f\left(x_{n}\right): n \in \omega\right\}$ is a discrete set. Now using continuity of $f$, we easily find $\sigma_{s * n}, n \in \omega$, so that $x_{n} \in N_{\sigma_{s * n}}, \operatorname{diam}\left(f\left[N_{\sigma_{s * n}}\right]\right) \leq$ $1 /(l+2), \ln \left(\sigma_{s * n}\right)>l$, and $\left\{f\left[N_{\sigma_{s * n}}\right]: n \in \omega\right\}$ is discrete.

Put $H_{2}=\left\{x \in \omega^{\omega}: \exists^{\infty} s x \mid \operatorname{lh}\left(\sigma_{s}\right)=\sigma_{s}\right\}$.

Proof of Theorem 3.1. Let $\mathcal{F}$ be the family of all closed sets $F \subset X$ such that $f \mid F$ is continuous. It follows from [S, Theorem 1] that either $X$ can be covered by countably many members of $\mathcal{F}$, i.e., we get (i), or there is $X^{\prime} \subset X$ which is Polish in the relative topology and $X^{\prime}$ cannot be covered by countably many sets from $\mathcal{F}$. Thus, we can assume that $X$ is Polish and that (i) fails.

By a transfinite derivation process, we produce an ordinal $\alpha<\omega_{1}$ and a descending transfinite sequence of closed sets $F_{\xi}, \xi<\alpha$, so that

1) $f \mid\left(\bigcap_{\gamma<\xi} F_{\gamma} \backslash F_{\xi}\right)$ is continuous for all $\xi<\alpha$;

2) the set of discontinuity points of $f \mid F$ is dense in $F$, where $F=\bigcap_{\xi<\alpha} F_{\xi}$.

Case 1. $F=\emptyset$.

Then since $\bigcap_{\gamma<\xi} F_{\gamma} \backslash F_{\xi}$ is $F_{\sigma}$, we can easily find countably many closed sets $X_{n}, n \in \omega$, so that $\bigcup_{n} X_{n}=X$ and $f \mid X_{n}$ is continuous, which contradicts our assumption. 
Case 2. $F \neq \emptyset$.

Let $K \subset F$ and $D \subset K$ be as in Lemma 3.4. (We apply it to $f \mid F$.) We can assume that $X=K$. Since continuity points of $f$ constitute a dense $G_{\delta}$ and no point in $D$ is a continuity point of $f$, by Hurewicz's theorem, we can find an embedding $\phi_{1}: 2^{\omega} \rightarrow K$ so that $x \in Q \Rightarrow \phi_{1}(x) \in D$ and $x \notin Q \Rightarrow \phi_{1}(x)$ is a continuity point of $f$. Consider $g=f \circ \phi_{1} \mid\left(2^{\omega} \backslash Q\right) ; g$ is continuous. We identify $2^{\omega} \backslash Q$ with $\omega^{\omega}$. Then $H \subset \omega^{\omega}$ is non- $\sigma$-bounded iff there is no $G_{\delta}$ set $G$ such that $G \cap H=\emptyset$ and $Q \subset G$. Let $H \subset \omega^{\omega}$ be closed, non- $\sigma$-bounded such that either $g \mid H$ is constant or $g \mid H$ is an embedding (Lemma 3.5). Again by Hurewicz's theorem, there is an embedding $\phi_{2}: 2^{\omega} \rightarrow 2^{\omega}$ such that $x \in Q \Rightarrow \phi_{2}(x) \in Q$ and $x \notin Q \Rightarrow \phi_{2}(x) \in H$. Put $\phi=\phi_{1} \circ \phi_{2}$. Then clearly

a) $x \in Q \Rightarrow x$ is $f \circ \phi$-isolated;

b) $x \notin Q \Rightarrow x$ is a continuity point of $f \circ \phi$;

c) given $\epsilon>0, \operatorname{osc}(f \circ \phi, x)<\epsilon$ for all but finitely many $x \in Q$.

We now have two subcases.

Subcase 1. $f \circ \phi \mid\left(2^{\omega} \backslash Q\right)$ is constant.

Subcase 2. $f \circ \phi \mid\left(2^{\omega} \backslash Q\right)$ is an embedding.

An application of Lemma 3.2 in each of these subcases finishes the proof.

Remarks. 1. To derive [JR, Theorem 1] from Theorem 3.1 combine the following three obvious facts: (1) if $f: X \rightarrow Y$ is not Baire class 1, then there is an open, so $F_{\sigma}$, set $A \subset Y$ with $f^{-1}(A)$ not $F_{\sigma}$; (2) if $g \sqsubseteq f, g: X_{1} \rightarrow Y_{1}, f: X \rightarrow Y$, and there is an $F_{\sigma}$ set $B \subset Y_{1}$ with $g^{-1}(B)$ not $F_{\sigma}$, then there is an $F_{\sigma}$ set $A \subset Y$ with $f^{-1}(A)$ not $F_{\sigma} ;(3) L^{-1}\left(\left\{\sum_{n=0}^{\infty} x(n) / 3^{n+1}: x \in 2^{\omega}\right\}\right)$ and $L_{1}^{-1}(\{0\})$ are not $F_{\sigma}$.

2. One could ask if in Theorem 3.1 it is possible to have one function instead of two- $L$ and $L_{1}$ - that is, a function $F: Z_{1} \rightarrow Z_{2}, Z_{1}, Z_{2}$ metric separable, such that $\operatorname{dec}_{c}(F)>\omega$ and $F \sqsubseteq f$ for any Baire class 1 function $f: X \rightarrow Y$ with $\operatorname{dec}_{c}(f)>\omega$. This is however impossible. Indeed, for any such $F$, we would have $F \sqsubseteq L$ and $F \sqsubseteq L_{1}$. But $F \sqsubseteq L$ implies that $F$ is 1 -to- 1 while $F \sqsubseteq L_{1}$ implies that the range of $F$ is countable. It would follow that the domain of $F$ is countable which, in turn, would give $\operatorname{dec}_{c}(F) \leq \omega$.

3. Theorem 3.1 does not generalize to arbitrary Borel functions. Simply take $f: \mathbb{R} \rightarrow \mathbb{R}$ to be equal to 0 on $\mathbb{Q}$ and 1 on $\mathbb{R} \backslash \mathbb{Q}$. Then obviously neither $L \sqsubseteq f$ nor $L_{1} \sqsubseteq f$, and it is easy to see that $\operatorname{dec}_{c}(f)>\omega$. However, in a conversation with $\mathrm{K}$. Kunen we convinced ourselves that for an arbitrary $f: X \rightarrow Y, X$ Souslin, either $\operatorname{dec}_{c}(f) \leq \omega$ or $\operatorname{dec}_{c}(f) \geq \mathfrak{d}$ (compare Corollary 3.7 below).

The first part of the following proposition is due to Cichon and Morayne. We include its proof here for the sake of completness. It was also known to Morayne that there is a Baire class 1 function $f$ with $\operatorname{dec}_{c}(f)=\mathfrak{d}$.

Proposition 3.6. (i) $[\mathrm{CM}]$ Let $f: X \rightarrow Y$ be Baire class 1, $X$ Souslin. Then $\operatorname{dec}_{c}(f) \leq \mathfrak{d}$.

(ii) $\operatorname{dec}_{c}\left(L_{1}\right)=\operatorname{dec}_{c}(L)=\mathfrak{d}$.

Proof. (i) [CM] Let $\pi: X \times Y \rightarrow X$ be the projection. The graph of $f$ is Souslin, so there is $\phi: \omega^{\omega} \rightarrow f \subset X \times Y$ continuous and onto. For any $x \in \omega^{\omega}, K_{x}=\{y \in$ $\left.\omega^{\omega}: \forall n y(n) \leq x(n)\right\}$ is compact, whence so is $\phi\left[K_{x}\right]$. Thus $\phi\left[K_{x}\right]$ is a graph of a continuous function defined on $\pi\left[\phi\left[K_{x}\right]\right]$ which is also compact, whence closed in $X$. Also, clearly $X=\bigcup_{x \in D} \pi\left[\phi\left[K_{x}\right]\right]$ for any dominating set $D \subset \omega^{\omega}$. 
(ii) The inequality $\leq$ follows from (i). To see $\geq$, note that if $L \mid F$ is continuous, $F \subset 2^{\omega}$ closed, then each point in $Q \cap F$ is isolated in $F$. Thus $F \backslash Q$ is still closed in $2^{\omega}$, whence it is compact. Thus if $\bigcup \mathcal{F}=2^{\omega}$ and, for any $F \in \mathcal{F}, F$ is closed and $L \mid F$ is continuous, then $\bigcup\{F \backslash Q: F \in \mathcal{F}\}=2^{\omega} \backslash Q$ and each $F \backslash Q$ is compact. Since $2^{\omega} \backslash Q$ is homeomorphic to $\omega^{\omega}$ and any compact subset of $\omega^{\omega}$ is bounded, we get $|\mathcal{F}| \geq \mathfrak{d}$. The proof for $L_{1}$ is similar.

Corollary 3.7. Let $f: X \rightarrow Y$ be Baire class $1, X$ Souslin. Then $\operatorname{dec}_{c}(f) \leq \omega$ or $\operatorname{dec}_{c}(f)=\mathfrak{d}$.

Proof. If (i) of Theorem 3.1 holds, then $\operatorname{dec}_{c}(f) \leq \omega$. If (ii) holds, then $\operatorname{dec}_{c}(f) \geq$ $\operatorname{dec}_{c}(L)$ or $\operatorname{dec}_{c}(f) \geq \operatorname{dec}_{c}\left(L_{1}\right)$; thus $\operatorname{dec}_{c}(f)=\mathfrak{d}$ by Proposition 3.6.

\section{DeComposing Baire Class 1 FUnCtions INTO CONTINUOUS FUNCTIONS WITH ARBITRARY DOMAINS}

In this section, we prove the second dichotomy theorem for Baire class 1 functions.

Theorem 4.1. Let $f: X \rightarrow Y$ be Baire class 1, $X$ Souslin. Then either there are $X_{n} \subset X, n \in \omega$, such that $\bigcup_{n} X_{n}=X$ and $f \mid X_{n}$ are continuous (i.e., $\left.\operatorname{dec}(f) \leq \omega\right)$, or $P \sqsubseteq f$.

Most of the proof of Theorem 4.1 consists of showing preparatory results to establish two main lemmas: 4.6 and 4.7. Lemma 4.6 shows that if $\operatorname{dec}(f)>\omega$, then the restriction of $f$ to a subset $Z$ of $X$ has three characteristic properties of $P$. (It is not difficult to check that $P$ satisfies properties (i)-(iii) from Lemma 4.6.) Lemma 4.7 then shows that $P$ is contained in $f \mid Z$.

Let $\widetilde{X}, \widetilde{Y}$ be Polish with $X \subset \widetilde{X}$ and $Y \subset \widetilde{Y}$. It is well known that $f$ can be extended to a Borel function $\tilde{f}: \widetilde{X} \rightarrow \widetilde{Y}$. Assume in the rest of this section that $\widetilde{X}$ and $\widetilde{Y}$ are recursively presented Polish spaces $X \in \Sigma_{1}^{1}$ and $\widetilde{f} \in \Delta_{1}^{1}$.

Lemma 4.2. Either $\operatorname{dec}(f) \leq \omega$, or there is a $\Sigma_{1}^{1}$ set $\emptyset \neq A \subset X$ such that $f \mid B$ is not continuous for any $\Sigma_{1}^{1}$ set $\emptyset \neq B \subset A$.

Proof. This lemma is, in a sense, a first level analog of Lemma 2.1; its original proof was a simplified version of that of Lemma 2.1. The usage of reflection was suggested to me by G. Hjorth. Let $P=\left\{C \subset \widetilde{X}: C \in \Sigma_{1}^{1}\right.$ and $\widetilde{f} \mid C$ is continuous $\}$. By reflection, for $C \in \Sigma_{1}^{1}$ with $\widetilde{f} \mid C$ continuous, there is a $C^{\prime} \in \Delta_{1}^{1}$ such that $C \subset C^{\prime}$ and $\widetilde{f} \mid C^{\prime}$ is continuous. Thus, $x \in \bigcup P$ iff $\exists C^{\prime} \in \Delta_{1}^{1}$ and $\widetilde{f} \mid C^{\prime}$ is continuous. Therefore, $\bigcup P \in \Pi_{1}^{1}$. If $X \subset \bigcup P$, clearly $\operatorname{dec}(f) \leq \omega$. If $X \not \subset \bigcup P$, put $A=X \backslash \bigcup P$.

A set $Z \subset X$ is called singular if there is an open set $U \subset Y$ such that $f^{-1}(U) \cap Z$ is nonempty, closed, and nowhere dense in $Z$.

Lemma 4.3. Let $\emptyset \neq A \subset X$ be $\Sigma_{1}^{1}$. Then either

(i) $\exists B \subset A B \in \Sigma_{1}^{1}, B \neq \emptyset$, and $f \mid B$ continuous, or

(ii) $\forall B \subset A, \emptyset \neq B \in \Sigma_{1}^{1}, \exists C \subset B C$ singular and $\Sigma_{1}^{1}$.

Proof. Assume that for some $\emptyset \neq B \subset A, B \in \Sigma_{1}^{1}$, the following holds:

$$
\forall C \subset B, C \in \Sigma_{1}^{1} \forall V \subset Y \text { open } f^{-1}(V) \cap C \neq \emptyset \Rightarrow \operatorname{int}_{C}\left(f^{-1}(V) \cap C\right) \neq \emptyset .
$$


We prove that $(*)$ implies that $f \mid B$ is continuous. Let $V \subset Y$ be basic open. Put

$$
C=\left\{x \in B: \forall W \subset X \text { basic open }\left(x \notin W \text { or } \exists z \in W z \in B \backslash f^{-1}(V)\right)\right\} .
$$

Then $C \in \Sigma_{1}^{1}$ and $C=B \backslash \operatorname{int}_{B}\left(f^{-1}(V) \cap B\right)$. If $f^{-1}(V) \cap C=\emptyset, f^{-1}(V) \cap B$ is open in $B$. So assume $f^{-1}(V) \cap C \neq \emptyset$. Then by $(*)$ there is $W \subset X$ basic open such that $\emptyset \neq W \cap C \subset f^{-1}(V) \cap C$. But then $W \cap B \subset \operatorname{int}_{B}\left(f^{-1}(V) \cap B\right)$, whence $W \cap C=\emptyset$, a contradiction.

Now, assume that for all $\emptyset \neq B \subset A, B \in \Sigma_{1}^{1}$, we have $\neg(*)$. We show that (ii) holds. Thus, let $\emptyset \neq B \subset A, B \in \Sigma_{1}^{1}$. Pick $C_{1} \subset B, C_{1} \in \Sigma_{1}^{1}$, and $V \subset Y$ basic open such that $f^{-1}(V) \cap C_{1} \neq \emptyset$ and $\operatorname{int}_{C_{1}}\left(f^{-1}(V) \cap C_{1}\right)=\emptyset$. Note that $f^{-1}(V)$ is an $F_{\sigma}$ and $f^{-1}(V) \cap C_{1} \in \Sigma_{1}^{1}$. Since $f^{-1}(V) \cap C_{1}$ with the Gandy-Harrington topology is a Baire space, there is $\emptyset \neq C_{2} \subset f^{-1}(V) \cap C_{1}, C_{2} \in \Sigma_{1}^{1}$, and $\bar{C}_{2} \subset f^{-1}(V)$. Thus, $C_{2}$ is closed and nowhere dense in $C=C_{2} \cup\left(C_{1} \backslash f^{-1}(V)\right)$. Also, $f^{-1}(V) \cap C=C_{2}$. Thus $C$ is singular and $\Sigma_{1}^{1}$.

Lemma 4.4. Let $\emptyset \neq D_{n} \subset \cdots \subset D_{1} \subset A \subset X$ be all open in the GandyHarrington topology with $D_{1}$ closed nowhere dense in $A$. Assume $S_{1}, S_{2} \subset A \backslash D_{1}$ are disjoint and such that $A \backslash S_{1}$ and $A \backslash S_{2}$ are open in the Gandy-Harrington topology. Then there are $i_{0} \in\{1,2\}$ and an open, in the Gandy-Harrington topology, set $A^{\prime} \subset A$ such that

(i) $A^{\prime} \cap D_{n} \neq \emptyset$;

(ii) $A^{\prime} \cap D_{1}$ is nowhere dense in $A^{\prime}$;

(iii) if $D_{i+1}$ is nowhere dense in $D_{i}$, then $A^{\prime} \cap D_{i+1}$ is nowhere dense in $A^{\prime} \cap D_{i}$;

(iv) $A^{\prime} \cap S_{i_{0}}=\emptyset$.

Proof. We start by formulating a claim.

Claim. There are $i_{0} \in\{1,2\}$ and relatively open sets $\emptyset \neq W_{i} \subset D_{i}, 1 \leq i \leq n$, such that

1) $\bigcup_{1<i<n} W_{i} \subset \overline{A \backslash\left(S_{i_{0}} \cup D_{1}\right)}$

2) $\forall 1 \leq j \leq n\left(\bigcup_{1 \leq i \leq j} W_{i}\right) \cap D_{j}$ is dense in $\left(\bigcup_{1 \leq i \leq n} W_{i}\right) \cap D_{j}$.

Assuming the claim has been proved, put

$$
A^{\prime}=\bigcup_{1 \leq i \leq n} W_{i} \cup\left(A \backslash\left(S_{i_{0}} \cup D_{1}\right)\right) .
$$

It is clear that (i), (ii), and (iv) hold. To see (iii), note that for any $1 \leq j \leq n$, $A^{\prime} \cap D_{j}$ contains a dense subset relatively open in $D_{j}$, namely $\left(\bigcup_{1 \leq i \leq j} W_{i}\right) \cap D_{j}$. (Density follows from 2).)

Thus, it is enough to prove the claim. Put $Z_{i}=A \backslash\left(S_{i} \cup D_{1}\right), i=1,2$. The claim will follow, if we show that there are $i_{0} \in\{1,2\}$ and relatively open sets $\emptyset \neq W_{i} \subset D_{i}, 1 \leq i \leq n, \emptyset \neq W_{0} \subset A$ such that

a) $Z_{i_{0}} \cap W_{0}$ is dense in $\bigcup_{i \leq n} W_{i}$;

b) $\forall 1 \leq j \leq n\left(\bigcup_{1 \leq i \leq j} W_{i}\right) \cap D_{j}$ is dense in $\left(\bigcup_{1 \leq i \leq n} W_{i}\right) \cap D_{j}$.

For $Z \subset A$ put $Z^{0}=\operatorname{int}_{A}(\bar{Z})$ and $Z^{j+1}=\operatorname{int}_{D_{j+1}}\left(\overline{Z^{j}}\right), j<n$. Note first that $Z_{1}^{j} \cup Z_{2}^{j}$ is dense in $D_{j}$ for any $j \leq n$. This is proved by induction: since $\bar{Z}_{1} \cup \bar{Z}_{2}=\bar{Z}_{1} \cup Z_{2} \supset A, Z_{1}^{0} \cup Z_{2}^{0}=\operatorname{int}_{A} \bar{Z}_{1} \cup \operatorname{int}_{A} \bar{Z}_{2}$ is dense in $A$. A similar argument shows that denseness of $Z_{1}^{j} \cup Z_{2}^{j}$ in $D_{j}$ implies denseness of $Z_{1}^{j+1} \cup Z_{2}^{j+1}$ in $D_{j+1}$. Note also that if $Z_{i}^{j}=\emptyset$, then $Z_{i}^{j+1}=\emptyset$. Since $Z_{1}^{n} \cup Z_{2}^{n}$ is dense in $D_{n}$, there is $i_{0} \in\{1,2\}$ such that $Z_{i_{0}}^{n} \neq \emptyset$; thus $Z_{i_{0}}^{j} \neq \emptyset$ for any $j \leq n$. Put $W_{j}=Z_{i_{0}}^{j}$. 
Obviously $W_{j}$ is relatively open in $D_{j}$. It is also clear that $W_{0}=Z_{i_{0}}^{0}$ is dense in $\bigcup_{i \leq n} W_{i}$ and $Z_{i_{0}} \cap Z_{i_{0}}^{0}$ is dense in $Z_{i_{0}}^{0}$; whence $Z_{i_{0}} \cap W_{0}$ is dense in $\bigcup_{i \leq n} W_{i}$. To see b), note that $W_{j} \subset D_{j}$ and $W_{j}$ is dense in $\bigcup_{j \leq i \leq n} W_{i}$. Since obviously $\left(\bigcup_{1 \leq i<j} W_{i}\right) \cap D_{j}$ is dense in $\left(\bigcup_{1 \leq i<j} W_{i}\right) \cap D_{j}$, we get $\left.\mathrm{b}\right)$ which finishes the proof of the claim.

Lemma 4.5. Assume $f$ is not constant on any set open in the Gandy-Harrington topology. Let $Y \supset U_{1} \supset U_{2} \supset \cdots \supset U_{n}$ be basic open, and let $A$ be open in the Gandy-Harrington topology. Assume that $f^{-1}\left(U_{n}\right) \cap A \neq \emptyset$ and that $f^{-1}\left(U_{1}\right) \cap A$ is closed and nowhere dense in $A$. For $i \leq m$, let $V^{i} \subset A$ be relatively open, and let $V_{i} \subset Y$ be open with $V^{i} \cap f^{-1}\left(V_{i}\right) \neq \emptyset$ and $V_{i} \cap U_{1}=\emptyset$. Then there are basic open sets $O_{i} \subset V_{i}, i \leq m$, and a set $A^{\prime} \subset A$ open in the Gandy-Harrington topology such that

(i) $\bar{O}_{i_{1}} \cap \bar{O}_{i_{2}}=\emptyset$ if $i_{1} \neq i_{2}$;

(ii) $\emptyset \neq f^{-1}\left(O_{i}\right) \cap A^{\prime} \subset V^{i}$;

(iii) $f^{-1}\left(U_{n}\right) \cap A^{\prime} \neq \emptyset$;

(iv) $f^{-1}\left(U_{1}\right) \cap A^{\prime}$ is closed and nowhere dense in $A^{\prime}$;

(v) if $f^{-1}\left(U_{j+1}\right) \cap A$ is nowhere dense in $f^{-1}\left(U_{j}\right) \cap A$, then $f^{-1}\left(U_{j+1}\right) \cap A^{\prime}$ is nowhere dense in $f^{-1}\left(U_{j}\right) \cap A^{\prime}$ for $j<n$.

Proof. First, note that since $f$ is not constant on any set open in the GandyHarrington topology, $f \mid\left(V^{i} \cap f^{-1}\left(V_{i}\right)\right), i \leq m$, attains infinitely many values. Thus by shrinking the $V_{i}$ 's, we can assure that $\bar{V}_{i_{1}} \cap \bar{V}_{i_{2}}=\emptyset$ if $i_{1} \neq i_{2}$ but still $V^{i} \cap$ $f^{-1}\left(V_{i}\right) \neq \emptyset$. Thus (i) will be fulfilled automatically as long as $O_{i} \subset V_{i}$.

Now by recursion on $i \leq m$, we will find $A_{i}^{\prime} \subset A$ open in the Gandy-Harrington topology and basic open sets $O_{i} \subset V_{i}$ such that (iii)-(v) hold for $A^{\prime}=A_{i}^{\prime}$ and

(vi) $f^{-1}\left(O_{i}\right) \cap V^{i} \neq \emptyset$,

(vii) $f^{-1}\left(O_{i}\right) \cap A_{i}^{\prime}=\emptyset$, and

(viii) $A_{i+1}^{\prime} \subset A_{i}^{\prime}$.

I will just show how to obtain $O_{0}$ and $A_{0}^{\prime}$ from $A$; one gets $O_{i+1}$ and $A_{i+1}^{\prime}$ from $A_{i}^{\prime}$ by the same argument. Since $f$ is not constant on $V^{0} \cap f^{-1}\left(V_{0}\right)$, there are $O^{1}, O^{2} \subset V_{0}$ open and such that $O^{1} \cap O^{2}=\emptyset$ and $f^{-1}\left(O^{i}\right) \cap V^{0} \neq \emptyset, i \in\{1,2\}$. Consider the sets

$$
A \supset f^{-1}\left(U_{1}\right) \cap A \supset \cdots \supset f^{-1}\left(U_{n}\right) \cap A,
$$

and

$$
S_{1}=f^{-1}\left(O^{1}\right) \cap A \text { and } S_{2}=f^{-1}\left(O^{2}\right) \cap A .
$$

Apply Lemma 4.4 to $D_{i}=f^{-1}\left(U_{i}\right) \cap A$ and $S_{1}, S_{2}$ to obtain $i_{0} \in\{1,2\}$ and $A^{\prime} \subset A$. Put $A_{0}^{\prime}=A^{\prime}$ and $O_{0}=O^{i_{0}}$. It is clear that (iii)-(viii) are fulfilled by these sets.

Having produced the $A_{i}^{\prime}$ 's and the $O_{i}$ 's, put

$$
A^{\prime}=A_{m}^{\prime} \cup \bigcup_{i \leq m}\left(f^{-1}\left(O_{i}\right) \cap V^{i}\right)
$$

Now, it is easy to check that $A^{\prime}$ along with the $O_{i}$ 's fulfil (i)-(v). Let us only mention that $f^{-1}\left(U_{1}\right) \cap A^{\prime}$ is closed in $A^{\prime}$, as claimed in (iv), since $f^{-1}\left(U_{1}\right) \cap A$ is closed in $A$ and $A^{\prime} \subset A$, and that the rest of the argument proving (iv) and (v) is based on the fact that if $B \cap C$ is nowhere dense in $C$, then $B \cap\left(C \cup C_{1}\right)$ is nowhere dense in $C \cup C_{1}$ provided $B \cap C_{1}=\emptyset$. (We apply this last fact to $C=A_{m}^{\prime}$ and $C_{1}=\bigcup_{i \leq m}\left(f^{-1}\left(O_{i}\right) \cap V^{i}\right)$.) 
Lemma 4.6. Assume $A \subset X$ is $\Sigma_{1}^{1}$ and (ii) of Lemma 4.3 holds. Then there is a set $Z \subset A$ such that

(i) $f[Z]$ is homeomorphic to $\omega^{\omega}$;

(ii) $f \mid Z: Z \rightarrow f[Z]$ is 1 -to-1 and open;

(iii) for any $\emptyset \neq U \subset f[Z]$ relatively open there is $\emptyset \neq V \subset U$ relatively open such that $(f \mid Z)^{-1}(V)$ is nowhere dense in $(f \mid Z)^{-1}(U)$.

Proof. Let us fix a winning strategy $\Sigma$ for $\underline{\alpha}$ in the Choquet game for $X$ with the Gandy-Harrington topology. (See [HKL] for details on the Choquet game for this topology.) Let $d$ be a totally bounded metric on $X$, and let $\rho$ be a complete metric on $Y$. Recursively with respect to $n \in \omega$, we define finite trees $T_{n} \subset \omega^{<\omega}$ so that

1) $\bigcup_{n} T_{n}=\omega^{<\omega}$

2) $T_{n} \subset T_{n+1}$;

3) if $\sigma * k \in T_{n}$, then $\sigma * l \in T_{n}$ for all $l<k$.

Additionally, we construct $A_{n} \subset X$ open in the Gandy-Harrington topology and $U_{\sigma} \subset Y, \sigma \in T_{n}$, basic open so that

4) $A_{n+1} \subset A_{n}$

5) $\rho-\operatorname{diam}\left(U_{\sigma}\right) \leq 1 /(\operatorname{lh}(\sigma)+1)$;

6) if $\sigma \subset \tau \in T_{n}$, then $U_{\tau} \subset U_{\sigma}$;

7) if $\sigma, \tau \in T_{n}$ and $\sigma$ and $\tau$ are incompatible, then $\bar{U}_{\sigma} \cap \bar{U}_{\tau}=\emptyset$;

8) if $\sigma * 0 \in T_{n}$, then $A_{n} \cap f^{-1}\left(U_{\sigma * 0}\right)$ is closed and nowhere dense in $A_{n} \cap$ $f^{-1}\left(U_{\sigma} \backslash \bigcup_{\sigma * k \in T_{n}, k \geq 1} \bar{U}_{\sigma * k}\right)$

9) $d-\operatorname{diam}\left(A_{n} \cap f^{-1}\left(U_{\sigma}\right)\right) \leq 1 /(\operatorname{lh}(\sigma)+1)$;

10) if $\sigma * k \in T_{n+1} \backslash T_{n}$ with $k \geq 1$, then for each $x \in A_{n+1} \cap f^{-1}\left(U_{\sigma * 0}\right)$ one can find $\sigma * m \in T_{n+1}$ with $m \geq 1$ and such that

$$
\forall y \in A_{n+1} \cap f^{-1}\left(U_{\sigma * m}\right) d(x, y)<1 /(n+1) ;
$$

11) let $\sigma \in T_{n}$ be terminal, and let $\sigma_{0} \subset \sigma_{1} \subset \cdots \subset \sigma_{n}=\sigma$ be such that $\sigma_{i}$ is terminal in $T_{i}, i \leq n$; then

$$
A_{n+1} \cap f^{-1}\left(U_{\sigma}\right) \subset \Sigma\left(A_{0} \cap f^{-1}\left(U_{\sigma_{0}}\right), \ldots, A_{n} \cap f^{-1}\left(U_{\sigma_{n}}\right)\right) .
$$

Let $\left\{\sigma_{n}: n \in \omega\right\}=\omega^{<\omega}$, and assume that $\forall \sigma \in \omega^{<\omega} \exists^{\infty} n \sigma=\sigma_{n}$. This will guarantee that 1) holds. Assume that $A_{n}, T_{n}$, and $U_{\sigma}, \sigma \in T_{n}$, have been constructed. First, we show that in the construction at the $n+1$ 'st stage we have to worry only about conditions 2)-10). Suppose we have carried out the construction up to stage $n$ maintaining 2)-11). Let $\sigma^{0}, \ldots, \sigma^{q}$ be the terminal nodes of $T_{n}$. For any $i \leq q$ and $j \leq n$, let $\sigma_{j}^{i} \subset \sigma^{i}$ be terminal in $T_{j}$. Define

$$
B_{i}=\Sigma\left(A_{0} \cap f^{-1}\left(U_{\sigma_{0}^{i}}\right), \ldots, A_{n} \cap f^{-1}\left(U_{\sigma_{n}^{i}}\right)\right),
$$

and

$$
A_{n}^{\prime}=\left(A_{n} \backslash \bigcup_{i \leq q} f^{-1}\left(U_{\sigma^{i}}\right)\right) \cup \bigcup_{i \leq q} B_{i} .
$$

Note that $A_{0}, \ldots, A_{n-1}, A_{n}^{\prime}, T_{0}, \ldots, T_{n-1}, T_{n}$, and $U_{\sigma}, \sigma \in T_{n}$, still fulfil 2)-11). Moreover, if we construct $A_{n+1} \subset A_{n}^{\prime}, T_{n+1}$, and $U_{\sigma}, \sigma \in T_{n+1}$, with properties 2)-10), they will automatically fulfil 11). Thus having constructed $A_{n}, T_{n}, U_{\sigma}$, $\sigma \in T_{n}$, with 2)-10), it is enough to find $A_{n+1}, T_{n+1}$, and $U_{\sigma}, \sigma \in T_{n+1}$, with $2)-10$ ), and this is what will be done below. 
Put $\sigma_{n}=\sigma$ and $\operatorname{lh}(\sigma)=l$.

Case 1. $\exists k<\sigma(l-1) \sigma \mid(l-1) * k \notin T_{n}$ or $\sigma \in T_{n}$ or $\sigma \mid(l-1) \notin T_{n}$.

We do not do anything, i.e., $T_{n+1}=T_{n}$ and $A_{n+1}=A_{n}$.

Case 2. $\sigma \notin T_{n}, \sigma \mid(l-1) \in T_{n}$, and $\sigma(l-1)=0$.

Put $T_{n+1}=T_{n} \cup\{\sigma\}$. Let $A \subset A_{n} \cap f^{-1}\left(U_{\sigma \mid(l-1)}\right)$ be $\Sigma_{1}^{1}$ and singular. Let $V \subset U_{\sigma \mid(l-1)}$ be open and such that $f^{-1}(V) \cap A$ is nonempty, closed in $A$, and nowhere dense in $A$. Let $U_{\sigma} \subset V$ be basic open such that $\rho-\operatorname{diam}\left(U_{\sigma}\right)<1 /(l+1)$, $f^{-1}\left(U_{\sigma}\right) \cap A \neq \emptyset$. Let $\emptyset \neq A^{\prime} \subset f^{-1}\left(U_{\sigma}\right) \cap A$ be $\Sigma_{1}^{1}$ such that $d-\operatorname{diam}\left(A^{\prime}\right)<1 /(l+1)$. Put

$$
A_{n+1}=\left(A_{n} \backslash f^{-1}\left(U_{\sigma \mid(l-1)}\right)\right) \cup\left(A \backslash f^{-1}(V)\right) \cup A^{\prime} .
$$

It is not difficult to check that 2)-10) hold.

Case 3. $\sigma \notin T_{n}, \sigma(l-1)>0$, and $\forall k<\sigma(l-1) \sigma \mid(l-1) * k \in T_{n}$.

Let $\bar{\sigma}=\sigma \mid(l-1)$ and $\sigma_{0}=\bar{\sigma} * 0$. Find relatively open, nonempty sets

$$
V^{0}, \ldots, V^{m} \subset A_{n} \cap f^{-1}\left(U_{\bar{\sigma}} \backslash \bigcup_{\bar{\sigma} * k \in T_{n}} \bar{U}_{\bar{\sigma} * k}\right)
$$

so that

$-\overline{V^{i}} \cap \overline{V^{j}}=\emptyset$ if $i \neq j$,

$-d-\operatorname{diam}\left(V^{i}\right) \leq 1 /(2(n+1))$, and

- $\forall x \in A_{n} \cap f^{-1}\left(U_{\sigma_{0}}\right) \exists i \leq m \forall y \in V^{i} d(x, y)<1 /(2(n+1))$.

This is possible by 8 ). Additionally, find

$$
V_{i} \subset U_{\bar{\sigma}} \backslash \bigcup_{\bar{\sigma} * k \in T_{n}} \bar{U}_{\bar{\sigma} * k}
$$

open with $f^{-1}\left(V_{i}\right) \cap V^{i} \neq \emptyset$ and $\rho-\operatorname{diam}\left(V_{i}\right)<1 /(l+2)$. Put

$$
T_{n+1}=T_{n} \cup\{\sigma, \bar{\sigma} *(\sigma(l-1)+1), \ldots, \bar{\sigma} *(\sigma(l-1)+m)\} .
$$

Let $T=\left\{\tau: \sigma_{0} * \tau \in T_{n}\right\} . T$ is a tree. Let $\tau_{0}, \ldots, \tau_{q}$ be all the terminal nodes in $T$. Let

$$
A=A_{n} \cap f^{-1}\left(U_{\bar{\sigma}} \backslash \bigcup_{\bar{\sigma} * k \in T_{n}, k \geq 1} \bar{U}_{\bar{\sigma} * k}\right) .
$$

For each $\tau_{j}$ consider the sets

$$
U_{\sigma_{0}} \supset U_{\sigma_{0} * \tau_{j} \mid 1} \supset \cdots \supset U_{\sigma_{0} * \tau_{j}}
$$

and

$$
A_{j}=A \backslash \bigcup\left\{f^{-1}\left(U_{\sigma_{0} * \tau}\right): \sigma_{0} * \tau \in T_{n} \text { with } \tau \text { and } \tau_{j} \text { incompatible }\right\} .
$$

Applying repeatedly Lemma 4.5 , we define recursively on $j \leq q$ basic open sets $O_{i}^{j+1} \subset O_{i}^{j} \subset V_{i}$ and sets $A_{j}^{\prime} \subset A_{j}$ open in the Gandy-Harrington topology so that (i)-(v) of Lemma 4.5 hold for $A=A_{j}, O_{i}=O_{i}^{j}$ and $A^{\prime}=A_{j}^{\prime}$. Finally, put $U_{\bar{\sigma} *(\sigma(l-1)+i)}=O_{i}^{q}, i \leq m$, and

$$
A_{n+1}=\bigcup_{j \leq q} A_{j}^{\prime} \cup\left(A_{n} \backslash f^{-1}\left(U_{\bar{\sigma}}\right)\right) \cup \bigcup_{\bar{\sigma} * k \in T_{n}, k \geq 1} f^{-1}\left(U_{\bar{\sigma} * k}\right) \cap A_{n} .
$$

If the $T_{n}$ 's are constructed, let

$$
G=\bigcap_{n} \bigcup_{\sigma \in \omega^{n}} U_{\sigma}=\bigcup_{\eta \in \omega^{\omega}} \bigcap_{n} U_{\eta \mid n} \text { and } Z=\bigcap_{n} A_{n} \cap f^{-1}(G) .
$$


By 5)-7), $G$ is homeomorphic to $\omega^{\omega}$. Let $\eta \in \omega^{\omega}$, and let $\sigma_{n} \subset \eta$ be terminal in $T_{n}$. Then, by 11) and 4), the following is a play in the Choquet game for the Gandy-Harrington topology:

$$
\begin{aligned}
A_{0} \cap f^{-1}\left(U_{\sigma_{0}}\right), \Sigma\left(A_{0} \cap f^{-1}\left(U_{\sigma_{0}}\right)\right), & \\
& A_{1} \cap f^{-1}\left(U_{\sigma_{1}}\right), \Sigma\left(A_{0} \cap f^{-1}\left(U_{\sigma_{0}}\right), A_{1} \cap f^{-1}\left(U_{\sigma_{1}}\right)\right), \ldots
\end{aligned}
$$

where $\underline{\beta}$ plays first, and $\underline{\alpha}$ responds by its winning strategy $\Sigma$. Thus, $\bigcap_{n} f^{-1}\left(U_{\sigma_{n}}\right) \cap$ $A_{n} \neq \bar{\emptyset}$, whence there is $x \in Z$ with $\{f(x)\}=\bigcap_{n} U_{\eta \mid n}$. By 9), such an $x$ is unique. Therefore, $f[Z]=G$, and $f \mid Z$ is 1-to-1. By 9), for any $x \in Z$ and $\epsilon>0$ there exists $R \subset Z$ such that $x \in R, d-\operatorname{diam}(R)<\epsilon$, and $f[R]$ is open in $G$. It follows that $f \mid Z: Z \rightarrow f[Z]$ is open. To see (iii), let $U \subset G$ be relatively open. Let $\sigma \in \omega^{<\omega}$ be such that $U_{\sigma} \cap G \subset U$. Then by 8), $f^{-1}\left(U_{\sigma * 0} \cap G\right)$ is closed in $f^{-1}\left(U_{\sigma} \cap G\right)$, whence by 7$)$ and 10$), f^{-1}\left(U_{\sigma * 0} \cap G\right)$ is nowhere dense in $f^{-1}\left(U_{\sigma} \cap G\right)$ so also in $f^{-1}(U)$.

Let $Z$ be as in Lemma 4.6. We want to show that $P \sqsubseteq f \mid Z$. If we put $F=$ $(f \mid Z)^{-1}$, this will follow from the next lemma.

Lemma 4.7. Assume $F: \omega^{\omega} \rightarrow Z$ is continuous, 1-to-1, onto, and for any $\emptyset \neq$ $U \subset \omega^{\omega}$ open there is $\emptyset \neq V \subset U$ open such that $F[V]$ is nowhere dense in $F[U]$. Then $P \sqsubseteq F^{-1}$.

We will deduce the above statement from Lemma 4.8. To formulate it we need several definitions. Let $d$ be a totally bounded metric on $Z$. Let us equip $\mathcal{F}(Z)$, the set of all closed nonempty subsets of $Z$, with the Hausdorff metric induced by $d$. We denote this metric also by $d$. Total boundedness of the metric $d$ on $Z$ implies the following fact which will be used repeatedly in the proofs below: given $\epsilon>0$ and $K \in \mathcal{F}(Z)$ there is a finite set $A \subset Z$ with $d(A, K)<\epsilon$. In the sequel, $\mathcal{F}(Z)$ is always considered as a topological space with the topology induced by $d$. Let $\Omega$ denote the set of all nonempty open subsets of $\omega^{\omega}$. For $\emptyset \neq U \subset \omega^{\omega}$ open, let $\Omega(U)$ be the set of all nonempty open subsets of $U$. Call a nonempty open subset of $\omega^{\omega}$ $n$-good, for $n \in \omega$, if it is a finite union of sets of the form $N_{\sigma}$ with $\sigma \in \omega^{m}, m \geq n$. A function $\phi: X \rightarrow \Omega$ is called $n$-good, $n \in \omega$, if $\phi(x)$ is $n$-good for any $x \in X$. For $\phi: X \rightarrow \Omega$ define $\phi^{F}: X \rightarrow \mathcal{F}(Z)$ by

$$
\phi^{F}(x)=\overline{F[\phi(x)]} .
$$

A function $\phi: X \rightarrow \Omega$ is called disjoint if $\phi^{F}\left(x_{1}\right) \cap \phi^{F}\left(x_{2}\right)=\emptyset$ for $x_{1} \neq x_{2}$, $x_{1}, x_{2} \in X$; it is called continuous if $\phi^{F}$ is continuous.

Lemma 4.8. There is a sequence of functions $\phi_{n}:(\omega+1)^{n} \rightarrow \Omega, n \in \omega$, such that

(i) $d-\operatorname{diam}\left(\phi_{n}^{F}(\eta)\right) \leq 1 /(n+1), \eta \in(\omega+1)^{n}$;

(ii) $\phi_{n+1}(\eta) \subset \phi_{n}(\eta \mid n), \eta \in(\omega+1)^{n+1}$;

(iii) $\phi_{n}$ is $n$-good;

(iv) $\phi_{n}$ is disjoint;

(v) $\phi_{n}$ is continuous.

Proof of Lemma 4.7 from Lemma 4.8. Notice the following fact which is a simple consequence of König's lemma:

(*) Assume $U_{n} \subset \omega^{\omega}$ is $n$-good, $n \in \omega$, and $U_{n+1} \subset U_{n}$; then $\bigcap_{n} U_{n} \neq \emptyset$. 
So, in particular, by (ii) and (iii), $\bigcap_{n} \phi(\eta \mid n) \neq \emptyset$ for any $\eta \in(\omega+1)^{\omega}$, and by (i) and the fact that $F$ is 1-to-1,

$(* *) \bigcap_{n} \phi(\eta \mid n)$ has precisely one element.

Define $\phi:(\omega+1)^{\omega} \rightarrow Z$ by letting $\phi(\eta)$ be the unique element of $\bigcap_{n} \phi_{n}^{F}(\eta \mid n)=$ $F\left[\bigcap_{n} \phi_{n}(\eta \mid n)\right]$. Note that $\phi(\eta)$ is well defined for all $\eta \in(\omega+1)^{\omega}$ by $(* *)$. $\phi$ is continuous by (v), (i), and (ii) and 1-to-1 by (iv); thus, since $(\omega+1)^{\omega}$ is compact, $\phi$ is an embedding.

Put $G=\bigcap_{n} \bigcup_{\eta \in(\omega+1)^{n}} \phi_{n}(\eta)$, and define $\psi: G \rightarrow \omega^{\omega}$ as follows. Let $x \in G$. By (iv) and (ii), there is a unique $\eta \in(\omega+1)^{\omega}$ with $x \in \bigcap_{n} \phi_{n}(\eta \mid n)$. Let $\psi(x)=P(\eta)$. We claim that $\psi$ is an embedding, and that it is onto $\omega^{\omega}$. Continuity of $\psi$ is obvious. By $(* *)$ and the fact that $P$ is onto, $\psi$ is onto. To show that it is open, we have to find, for any $x \in G$ and $N_{\sigma}$ with $x \in N_{\sigma}$, an $n \in \omega$ such that $\phi_{n}(\eta \mid n) \subset N_{\sigma}$ where $\eta$ is the unique element of $(\omega+1)^{\omega}$ with $x \in \bigcap_{n} \phi_{n}(\eta \mid n)$. But if for infinitely many $n, \phi_{n}(\eta \mid n) \backslash N_{\sigma} \neq \emptyset$, then we apply $(*)$ to the family $\phi_{n}(\eta \mid n) \backslash N_{\sigma}$ for $n>l h(\sigma)$, which is legal by (iii), and obtain $y \in \bigcap_{n} \phi_{n}(\eta \mid n) \backslash N_{\sigma}$, which contradicts (**) since $x \neq y$.

Now, we claim that $\phi \circ P^{-1} \circ \psi=F \mid G$. Note first that for any $x \in G$ and the unique $\eta \in(\omega+1)^{\omega}$ with $x \in \bigcap_{n} \phi_{n}(\eta \mid n)$, we have

$$
\phi \circ P^{-1} \circ \psi(x)=\text { the unique element in } \bigcap_{n} \phi_{n}^{F}(\eta \mid n) \text {. }
$$

But for any $n, F(x) \in \phi_{n}^{F}(\eta \mid n)$; thus, by (i), $F(x)=\phi \circ P^{-1} \circ \psi(x)$. Now since $F, \phi, P$, and $\psi$ are all 1-to-1, and $\psi$ is onto, it follows that $\psi^{-1} \circ P=F^{-1} \circ \phi$. Since $\psi^{-1}$ and $\phi$ are embeddings, we get $P \sqsubseteq F^{-1}$.

To prove Lemma 4.8, we will need one more auxiliary fact.

Lemma 4.9. (i) Let $U \in \Omega, \delta>0$, and $n \in \omega$. There is $\phi: \omega+1 \rightarrow \Omega(U)$ disjoint, continuous, $n$-good, and such that $d-\operatorname{diam}\left(\phi^{F}(\alpha)\right)<\delta$ for any $\alpha \in \omega+1$.

(ii) Let $U, V \in \Omega$. Assume $d(\overline{F[U]}, \overline{F[V]}) \leq \epsilon, \epsilon>0$. Let $\phi: \omega+1 \rightarrow \Omega(U)$ be disjoint and continuous. Let $n \in \omega$. Then there is $\xi: \omega+1 \rightarrow \Omega(V)$ disjoint, continuous, n-good, and such that $d\left(\phi^{F}(\alpha), \xi^{F}(\alpha)\right) \leq 2 \epsilon$ for $\alpha \in \omega+1$.

Proof. (i) Let $V \subset U, V \in \Omega$, be such that $F[V]$ is nowhere dense in $F[U]$. Find $\sigma \in \omega^{m}$, for some $m \geq n$, such that $N_{\sigma} \subset V$ and $d-\operatorname{diam}\left(F\left[N_{\sigma}\right]\right)<\delta$. Put $\phi(\omega)=N_{\sigma}$. Since $\overline{F[\phi(\omega)]}$ is nowhere dense in $\overline{F[U]}$, there are $W_{i} \subset \overline{F[U]}, i \in \omega$, relatively open and such that $\overline{W_{i}} \cap \overline{W_{j}}=\emptyset$ if $i \neq j, \overline{W_{i}} \cap \overline{F[\phi(\omega)]}=\emptyset, \overline{W_{i}} \rightarrow \overline{F[\phi(\omega)]}$ and $d-\operatorname{diam}\left(W_{i}\right)<\delta$. For each $i$ find an $n$-good set $V_{i}$ so that $V_{i} \subset U \cap F^{-1}\left(W_{i}\right)$ and $d\left(\overline{F\left[V_{i}\right]}, \overline{W_{i}}\right)<1 /(i+1)$. Put $\phi(i)=V_{i}$.

(ii) This is a refinement of the argument proving (i). Find a finite set $A \subset F[V]$ such that $d\left(\phi^{F}(\omega), A\right)<(3 / 2) \epsilon$. We find an $n$-good set $W \subset V$ so that $F[W]$ is nowhere dense in $F[V]$ and $d(A, \overline{F[W]})<(1 / 2) \epsilon$. To this end, for any $x \in A$, let $\sigma_{x} \in \omega^{m}, m \geq n$, be such that $N_{\sigma_{x}} \subset V, d\left(\{x\}, F\left[N_{\sigma_{x}}\right]\right)<(1 / 2) \epsilon$, and $F\left[N_{\sigma_{x}}\right]$ is nowhere dense in $F[V]$. Then put $\xi(\omega)=\bigcup_{x \in A} N_{\sigma_{x}}$. Let $A_{i} \subset F[V]$ be finite such that $A_{i} \rightarrow \overline{F[\xi(\omega)]}$ and $A_{i} \cap \overline{F[\xi(\omega)]}=\emptyset$. This is possible since $\overline{F[\xi(\omega)]}$ is nowhere dense in $\overline{F[V]}$. Since $\phi$ is continuous, by modifying finitely many of the $A_{i}$ 's, we can assume that $d\left(A_{i}, \phi^{F}(i)\right)<(3 / 2) \epsilon$ for all $i \in \omega$. Now, since $\overline{F[\xi(\omega)]}$ is nowhere dense in $\overline{F[V]}$, using a technique similar to that used in constructing $\xi(\omega)$ above we can find sets $W_{i} \subset V$ which are $n$-good and such that $\overline{F\left[W_{i}\right]} \cap \overline{F\left[W_{j}\right]}=\emptyset$ if $i \neq j$, 
$d\left(A_{i}, \overline{F\left[W_{i}\right]}\right)<\epsilon /(2 i+2), \overline{F\left[W_{i}\right]} \cap \overline{F[\xi(\omega)]}=\emptyset$. Put $\xi(i)=W_{i}$ for $i \in \omega$. It is easy to check that $\xi$ is as required.

Proof of Lemma 4.8. For a metric space $X$, we write $X^{\prime}$ for the set of all nonisolated points of $X$. First we observe that the following general claim holds.

Claim 1. Let $X$ be compact. Let $\psi: X \rightarrow \Omega$ be disjoint and continuous, and let $\phi: X^{\prime} \times(\omega+1) \rightarrow \Omega$ be disjoint, continuous, $n$-good, $n \in \omega$, with $\phi(x, \alpha) \subset \psi(x)$ and $d-\operatorname{diam}(\phi(x, \alpha))<\delta, \delta>0$, for all $(x, \alpha) \in X^{\prime} \times(\omega+1)$. Then there is a $\widetilde{\phi}: X \times(\omega+1) \rightarrow \Omega$ which extends $\phi$ and has all the above mentioned properties of $\phi$ except that $\widetilde{\phi}(x, \alpha) \subset \psi(x)$ and $d-\operatorname{diam}(\widetilde{\phi}(x, \alpha))<\delta$ hold for all $(x, \alpha) \in X \times(\omega+1)$.

Proof of Claim 1. First, we define an extension $\widetilde{\phi}$ which satisfies all the required conditions except perhaps $d-\operatorname{diam}(\widetilde{\phi}(x, \alpha))<\delta$. Let $x \in X \backslash X^{\prime}$. Find $y_{x} \in$ $X^{\prime}$ such that $d\left(\psi^{F}(x), \psi^{F}\left(y_{x}\right)\right)$ is minimal among $d\left(\psi^{F}(x), \psi^{F}(y)\right)$ for $y \in X^{\prime}$. Consider $\phi\left(y_{x}, \cdot\right): \omega+1 \rightarrow \Omega\left(\psi\left(y_{x}\right)\right)$. By Lemma 4.9(ii), there is a $\xi_{x}: \omega+$ $1 \rightarrow \Omega(\psi(x))$ disjoint, continuous, $n$-good, and such that $d\left(\phi^{F}\left(y_{x}, \alpha\right), \xi_{x}(\alpha)\right) \leq$ $2 d\left(\psi^{F}(x), \psi^{F}\left(y_{x}\right)\right)$. Put

$$
\widetilde{\phi}(x, \alpha)=\xi_{x}(\alpha), \text { for } x \in X \backslash X^{\prime} \text { and } \alpha \in \omega+1 .
$$

If $x \in X^{\prime}$, we put $\widetilde{\phi}(x, \alpha)=\phi(x, \alpha)$. It is clear that $\widetilde{\phi}$ is $n$-good and that $\widetilde{\phi}(x, \alpha) \subset$ $\psi(x)$ for $(x, \alpha) \in X \times(\omega+1)$. Also, if $(x, \alpha) \neq\left(x^{\prime}, \alpha^{\prime}\right)$, then $\widetilde{\phi}^{F}(x, \alpha) \cap \widetilde{\phi}^{F}\left(x^{\prime}, \alpha^{\prime}\right)=\emptyset$. It is enough to check the continuity of $\widetilde{\phi}^{F}$ on sequences of the form $\left(x_{n}, \alpha_{n}\right) \rightarrow(y, \alpha)$ where $x_{n} \in X \backslash X^{\prime}, y \in X^{\prime}$, and $\alpha_{n}, \alpha \in \omega+1$. Let $y_{n}$ be the $y_{x_{n}} \in X^{\prime}$ used to define $\xi_{x_{n}}$. Then, by definition of $y_{n}$,

$$
d\left(\psi^{F}\left(x_{n}\right), \psi^{F}\left(y_{n}\right)\right) \leq d\left(\psi^{F}\left(x_{n}\right), \psi^{F}(y)\right) \rightarrow 0 .
$$

Hence, since $X$ is compact, $x_{n} \rightarrow y$, and $\psi^{F}$ is continuous and 1-to-1, $y_{n} \rightarrow y$. Thus,

$$
\phi^{F}\left(y_{n}, \alpha_{n}\right) \rightarrow \phi^{F}(y, \alpha)
$$

as $\phi^{F}$ is continuous on $X^{\prime} \times(\omega+1)$. On the other hand,

$$
d\left(\phi^{F}\left(y_{n}, \alpha_{n}\right), \widetilde{\phi}^{F}\left(x_{n}, \alpha_{n}\right)\right) \leq 2 d\left(\psi^{F}\left(x_{n}\right), \psi^{F}\left(y_{n}\right)\right) \rightarrow 0 .
$$

Thus by (1) and $(2), \widetilde{\phi}^{F}\left(x_{n}, \alpha_{n}\right) \rightarrow \phi^{F}(y, \alpha)$. To get $d-\operatorname{diam}(\widetilde{\phi}(x, \alpha))<\delta$, we modify $\widetilde{\phi}$ constructed above as follows. The set

$$
\left\{(x, \alpha) \in X \times(\omega+1): d-\operatorname{diam}\left(\widetilde{\phi}^{F}(x, \alpha)\right)<\delta\right\}
$$

is open and contains $X^{\prime} \times(\omega+1)$. Thus,

$$
\left\{(x, \alpha) \in X \times(\omega+1): d-\operatorname{diam}\left(\widetilde{\phi}^{F}(x, \alpha)\right) \geq \delta\right\}
$$

is contained in a set of the form $\left\{x_{1}, \ldots, x_{m}\right\} \times(\omega+1)$ where each $x_{i}$ is an isolated point in $X$. Therefore, it suffices to redefine $\widetilde{\phi}$ on each $\left\{x_{i}\right\} \times(\omega+1)$ separately so that $\widetilde{\phi}^{F}\left(x_{i}, \alpha\right) \subset \psi^{F}\left(x_{i}\right)$ and $d-\operatorname{diam}\left(\widetilde{\phi}^{F}\left(x_{i}, \alpha\right)\right)<\delta$, and this can be done by Lemma 4.9(i).

Claim 2. Let $\psi:(\omega+1)^{n} \rightarrow \Omega$ be disjoint and continuous. Then there exists $\phi:(\omega+1)^{n+1} \rightarrow \Omega$ disjoint, continuous, $(n+1)$-good with $\phi(\eta) \subset \psi(\eta \mid n)$ and $d-\operatorname{diam}\left(\phi^{F}(\eta)\right)<1 /(n+2)$ for any $\eta \in(\omega+1)^{n+1}$. 
Proof of Claim 2. Write $(\omega+1)^{n+1}$ as $(\omega+1)^{n} \times(\omega+1)$. Let $X=(\omega+1)^{n}$. Put $X^{(0)}=X$ and $X^{(k+1)}=\left(X^{(k)}\right)^{\prime}$. Then, $X^{(n)}=\{(\omega, \ldots, \omega)\}$. Define $\phi$ : $X^{(n)} \times(\omega+1) \rightarrow \Omega(\psi(\omega, \ldots, \omega))$ using Lemma 4.9(i) with $\delta=1 /(n+2)$. Using Claim 1 extend $\phi$ consecutively to $X^{(n-1)} \times(\omega+1), X^{(n-2)} \times(\omega+1), \ldots$, and finally to $X^{(0)} \times(\omega+1)=(\omega+1)^{n+1}$.

To construct $\phi_{n}$ as in the conclusion of Lemma 4.8, let $\phi_{0}$ be defined according to Lemma 4.9(i) with $\delta=1$. If $\phi_{n}$ is defined, we find $\phi_{n+1}$ by applying Claim 2 to $\psi=\phi_{n}$.

\section{Complete semicontinuous functions}

In this section, we study complete semicontinuous functions. The results obtained here will be used to prove that $\operatorname{dec}(P)$ is highest possible and as a consequence establish an analogue of Corollary 3.7 for the decomposition into functions with arbitrary domains.

It will be convenient to widen the range of applicability of the definition of semicontinuity to certain functions whose image is contained in a compact space equipped with a closed linear order. Let $K$ be a compact, metric space. Let $\preceq \subset K \times K$ be closed. Assume $\preceq$ linearly orders $K$. A function $f: X \rightarrow K$, $X$ a metric, separable space, is called lower semicontinuous (lsc) if $f^{-1}(\{y \in K$ : $y_{0} \preceq y$ and $\left.\left.y_{0} \neq y\right\}\right)$ is open for any $y_{0} \in K$. An lsc function $f: X \rightarrow K$ will be called $K$-lsc complete if for any $g: 2^{\omega} \rightarrow K$ lsc there is a continuous function $\phi: 2^{\omega} \rightarrow X$ such that $g=f \circ \phi$. If $K=[0,1]$ and $\preceq=\leq$, we simply say lsc complete. We define upper semicontinuous (usc) and $K$-usc complete functions in a similar fashion. Since for any compact, metric $K$ and any $\preceq$ closed linear order on $K$ there exists an embedding $h: K \rightarrow[0,1]$ such that $x \preceq y$ iff $h(x) \leq h(y)$, we always implicitly assume that $K$ is embedded in $[0,1]$ and $\preceq=\leq \mid K$. A ray is a subset of $K$ of the form $\left\{y \in K: y_{0} \leq y\right\}$ or $\left\{y \in K: y_{0} \leq y\right.$ and $\left.y_{0} \neq y\right\}$ for some $y_{0} \in K$. We adopt the notation $\left\{y \in K: y_{0} \leq y\right\}=\left[y_{0}, \infty\right)$ and $\left\{y \in K: y_{0} \leq y\right.$ and $\left.y_{0} \neq y\right\}=\left(y_{0}, \infty\right)$.

Theorem 5.1. Let $F: X \rightarrow K$ be lsc. Then $F$ is $K$-lsc complete if, and only if, there exists $D \subset X$ Polish in the relative topology and such that $F[D]=K$ and for any $U \subset D$ relatively open $F[U]$ is a ray.

Proof. $(\Rightarrow)$ It is enough to find $g: 2^{\omega} \rightarrow K$ lsc such that $g\left[2^{\omega}\right]=K$ and for any $U \subset 2^{\omega}$ open $g[U]$ is a ray, since then there is a continuous function $\phi: 2^{\omega} \rightarrow X$ such that $F \circ \phi=g$, and it is easy to check that $D=\phi\left[2^{\omega}\right]$ works. To define $g$, fix a nondecreasing surjection $h: 2^{\omega} \rightarrow K$. Define the function sup : $\left(2^{\omega}\right)^{\omega} \rightarrow 2^{\omega}$ by $\sup \left(\left(x_{n}\right)\right)=\sup _{n} x_{n}$. Finally, put $g=h \circ \sup$, and note that $\left(2^{\omega}\right)^{\omega}$ is homeomorphic to $2^{\omega}$.

$(\Leftarrow)$ Assume we have $D$ as above. First, we show that

$$
\forall y, z \in K\left(y<z \Rightarrow F^{-1}(y) \cap D \subset \overline{F^{-1}(z) \cap D}\right),
$$

then that (1) implies

$$
\begin{aligned}
& \exists G \subset X(G \text { Polish, zero-dimensional, and } \\
& \qquad \forall f: G \rightarrow K \text { lsc } f \cap F \neq \emptyset \text { (i.e., } \exists x \in G f(x)=F(x))),
\end{aligned}
$$

and, finally, that (2) implies $F$ is $K$-lsc complete. 
If (1) fails for some $y<z$, there is $U \subset D$ relatively open such that $U \cap F^{-1}(y) \neq$ $\emptyset$ and $U \cap F^{-1}(z)=\emptyset$, i.e., $y \in F[U]$ and $z \notin F[U]$, which contradicts the assumption that $F[U]$ is a ray.

To prove (2) from (1), let $Q \subset K$ be countable and such that $\forall y \in K \forall \epsilon>0 \exists z \epsilon$ $Q y-\epsilon<z \leq y$. Note that $\min K \in Q$. For each $y \in Q$, let $Q_{y} \subset F^{-1}(y) \cap D$ be countable and dense in $F^{-1}(y) \cap D$. Let $G$ be zero-dimensional, $\Pi_{2}^{0}$ subset of $D$ such that $\bigcup_{y \in Q} Q_{y} \subset G$. We show that $G$ works. Let $G=\bigcap_{n} G_{n}, G_{n}$ open and $G_{n+1} \subset G_{n}$. Let $f: G \rightarrow K$ be lsc. Note that $Q \subset F[G]$ and for any $y, z \in Q$ with $y<z$ we have $F^{-1}(y) \cap G \subset \overline{F^{-1}(z) \cap G}$. This last condition implies that if $V$ is open and $y \in F[V]$, then $z \in F[V]$ for $y, z \in Q, y<z$. We recursively construct a sequence of open sets $U_{n}$ and $z_{n} \in Q, n \in \omega$, such that:

(i) $U_{n} \subset G_{n}$;

(ii) $\bar{U}_{n+1} \subset U_{n}$;

(iii) $\operatorname{diam}\left(U_{n}\right)<1 /(n+1)$;

(iv) $z_{n} \leq \inf f\left[U_{n}\right]$, and $z_{n} \in F\left[U_{n}\right]$;

(v) $\inf f\left[U_{n}\right]-1 /(n+1)<\inf F\left[U_{n+1}\right] \leq \inf f\left[U_{n}\right]$.

Let $U_{0}$ be open such that $\operatorname{diam}\left(U_{0}\right)<1, U_{0} \subset G_{0}$, and $\min K \in F\left[U_{0}\right]$. If $U_{n}$ has been defined, find $z_{n+1} \in Q$ with inf $f\left[U_{n}\right]-1 /(n+1)<z_{n+1} \leq \inf f\left[U_{n}\right]$ and $z_{n} \leq z_{n+1}$. Such a $z_{n+1}$ exists by the definition of $Q$ and by (iv). Since $z_{n} \in F\left[U_{n}\right]$, $z_{n+1} \in F\left[U_{n}\right]$. Since $F$ is lsc, there is $V \subset U_{n}$ open such that $z_{n+1} \in F[V]$ and $\inf f\left[U_{n}\right]-1 /(n+1)<\inf F[V]$. We get $U_{n+1}$ by making $V$ small enough. Now, let $x$ be the only element in $\bigcap_{n} U_{n}$. Then $x \in G$, and since $f$ and $F$ are lsc, by (v), we get

$$
f(x)=\sup _{n} \inf f\left[U_{n}\right]=\sup _{n} \inf F\left[U_{n}\right]=F(x) .
$$

Now we show that (2) implies that $F$ is $K$-lsc complete. We can assume that $G$ is a closed subset of $\omega^{\omega}$ so that $G=$ the set of all branches of $T$, for some tree $T \subset \omega^{<\omega}$. Let $f: 2^{\omega} \rightarrow K$ be lsc. We show that there is a continuous function $\phi: 2^{\omega} \rightarrow G$ such that $f=F \circ \phi$. We play the following game: Players I and II play interchangeably; I plays $x_{n} \in 2$, II plays $y_{n} \in \omega$ so that $\left(y_{0}, \ldots, y_{n}\right) \in T$; I wins iff $f\left(\left(x_{n}\right)\right) \neq F\left(\left(y_{n}\right)\right)$. By Martin's theorem, the game is determined. A winning strategy for I induces a continuous function $\psi: G \rightarrow 2^{\omega}$ such that $f \circ \psi \cap F=\emptyset$, which contradicts $(2)$ since $f \circ \psi$ is lsc. Therefore, II has a winning strategy. It induces a continuous function $\phi: 2^{\omega} \rightarrow G$ such that $f=F \circ \phi$.

Remark. Obviously, an analogous characterization of $K$-usc complete functions is true. We will be using it in section 7 .

Now, we present a construction of a family of Baire class 1 functions. These functions will be used in the proof of the existence of "minimal" lsc complete functions and in the proof that the decomposition coefficient of Pawlikowski's function is highest possible. Let $\preceq_{n} \subset 2^{n}, n \in \omega$, be partial orders. Assume that for $\sigma, \tau \in 2^{n+1}$

$$
\sigma \preceq_{n+1} \tau \Rightarrow \sigma\left|n \preceq_{n} \tau\right| n .
$$

Define $T_{\left(\preceq_{n}\right)} \subset \prod_{n} 2^{n}$ by

$$
x \in T_{\left(\preceq_{n}\right)} \text { iff } \forall n x(n) \preceq_{n} x(n+1) \mid n .
$$

Let $F_{\left(\preceq_{n}\right)}: T_{\left(\preceq_{n}\right)} \rightarrow 2^{\omega}$ be defined by

$$
F_{\left(\preceq_{n}\right)}(x)=\text { the unique } y \in 2^{\omega} \text { with } \forall n \forall^{\infty} k y|n=x(k)| n \text {. }
$$


Define a partial order $\preceq$ on $2^{\omega}$ by

$$
x \preceq y \text { iff } \forall n x\left|n \preceq_{n} y\right| n .
$$

Lemma 5.2. $F_{\left(\preceq_{n}\right)}$ is Baire class 1 and onto.

Proof. It is clear that $F_{\left(\preceq_{n}\right)}$ is a pointwise limit of a sequence of continuous functions, whence it is Baire class 1. For $y \in 2^{\omega}$ define $x \in T_{\left(\preceq_{n}\right)}$ by $x(n)=y \mid n, n \in \omega$. Then $F_{\left(\preceq_{n}\right)}(x)=y$. Thus $F_{\left(\preceq_{n}\right)}$ is onto.

Lemma 5.3. If $C \subset 2^{\omega}$ is closed and linearly ordered by $\preceq$, then $F_{\left(\preceq_{n}\right)} \mid F_{\left(\preceq_{n}\right)}^{-1}(C)$ is $C$-lsc complete.

Proof. By Lemma 5.2, $G=F_{\left(\swarrow_{n}\right)}^{-1}(C)$ is $\Pi_{2}^{0}$. Therefore, to check that $F_{\left(\swarrow_{n}\right)} \mid G$ is $C$-lsc complete, it is enough, by Theorem 5.1, to show that $F_{\left(\preceq_{n}\right)} \mid G$ is lsc and that for any $U \subset G$ relatively open $F_{\left(\preceq_{n}\right)}[U]$ is a ray with respect to $\preceq \mid C$. To this end, it is enough to see that

(i) if $U \subset T_{\left(\preceq_{n}\right)}$ is open, $y \in F_{\left(\preceq_{n}\right)}[U]$, and $y \preceq z$, then $z \in F_{\left(\preceq_{n}\right)}[U]$, and

(ii) if $x \in T_{\left(\preceq_{n}\right)}$ and $y \preceq F_{\left(\preceq_{n}\right)}(x), y \neq F_{\left(\preceq_{n}\right)}(x)$, then there is an open set $U \subset T_{\left(\preceq_{n}\right)}$ such that $x \in U$ and if $z \preceq y$, then $z \notin F_{\left(\preceq_{n}\right)}[U]$.

To see (i), find $x \in U$ with $F_{\left(\preceq_{n}\right)}(x)=y$. Fix $n \in \omega$ such that if $x^{\prime}(i)=x(i)$ for $i \leq n$, then $x^{\prime} \in U$. Define $\bar{x}$ so that $\bar{x}(i)=x(i)$ for $i \leq n$, and $\bar{x}(i)=z \mid i$ for $i>n$. It is easy to check that $\bar{x} \in T_{\left(\preceq_{n}\right)}$, and clearly $\bar{x} \in U$ and $F_{\left(\preceq_{n}\right)}(\bar{x})=z$. To see (ii), note that there is an $n \in \omega$ such that $x(n) \npreceq_{n} y \mid n$. Then $U=\left\{x^{\prime} \in T_{\left(\preceq_{n}\right)}\right.$ : $\left.x^{\prime}(n)=x(n)\right\}$ works.

Lemma 5.4. Assume there is a closed uncountable, linearly ordered by $\preceq$ subset of $2^{\omega}$. Then $\operatorname{dec}\left(F_{\left(\preceq_{n}\right)}\right) \geq \operatorname{dec}(f)$ for any lsc $f: 2^{\omega} \rightarrow[0,1]$.

Proof. Let $C \subset 2^{\omega}$ be closed, uncountable, linearly ordered by $\preceq$. We can easily find a copy $C_{0}$ of $2^{\omega}$ inside $C$ such that the lexicographic order is equal to $\preceq$ on $C_{0}$. Let $\phi: C_{0} \rightarrow[0,1]$ be an increasing homeomorphism, e.g., the Cantor function. By Lemma $5.3, F_{\left(\preceq_{n}\right)}[U]$ is a ray in $\left(C_{0}, \preceq\right)$ for any relatively open $U \subset F_{\left(\preceq_{n}\right)}^{-1}\left(C_{0}\right)$. Thus $\phi \circ F_{\left(\preceq_{n}\right)}[U]$ is a ray in $[0,1]$. It follows that $\phi \circ F_{\left(\preceq_{n}\right)} \mid F_{\left(\preceq_{n}\right)}^{-1}\left(C_{0}\right)$ is lsc complete. Thus, $\operatorname{dec}\left(\phi \circ F_{\left(\swarrow_{n}\right)}\right) \geq \operatorname{dec}(f)$ for any $f: 2^{\omega} \rightarrow[0,1]$ lsc. But since $\phi$ is continuous, $\operatorname{dec}\left(F_{\left(\preceq_{n}\right)}\right) \geq \operatorname{dec}\left(\phi \circ F_{\left(\swarrow_{n}\right)}\right)$.

$\sigma \in 2^{n}$ is called splitting if $\sigma * 0 \preceq_{n+1} \sigma * 1$, or $\sigma * 1 \preceq_{n+1} \sigma * 0$.

Lemma 5.5. Assume that for each $n \in \omega$ and any $\sigma, \tau \in 2^{n}$ with $\sigma \preceq_{n} \tau$ we have

$$
\forall i \in 2 \exists j \in 2 \quad \sigma * i \preceq_{n+1} \tau * j \text { and } \forall i \in 2 \exists j \in 2 \quad \sigma * j \preceq_{n+1} \tau * i .
$$

Assume also that for any $\sigma \in 2^{<\omega}$ there is a splitting $\tau \in 2^{<\omega}$ with $\sigma \subset \tau$. Then there is a perfect, closed set linearly ordered by $\preceq$.

Proof. The conclusion will follow easily if we can show that if $\sigma_{0}, \ldots, \sigma_{k} \in 2^{n}$, $\sigma_{0} \preceq_{n} \cdots \preceq_{n} \sigma_{k}$, and $i \leq k$, then there are $\tau_{0}, \ldots, \tau_{k+1} \in 2^{m}$ for some $m>n$ with $\tau_{j} \mid n=\sigma_{j}$ for $j \leq i$ and $\tau_{j} \mid n=\sigma_{j-1}$ for $j>i, \tau_{0} \preceq_{m} \cdots \preceq_{m} \tau_{k+1}$, and $\tau_{i} \neq \tau_{i+1}$. To see this, let $\tau \supset \sigma_{i}$ be splitting. Assume $\tau * 0 \preceq_{m} \tau * 1$ where $m=\operatorname{lh}(\tau * 0)$. Put $\tau_{i}=\tau * 0$ and $\tau_{i+1}=\tau * 1$. By (4), we can extend $\sigma_{i+1}, \ldots, \sigma_{k}$ one by one to $\tau_{i+2}, \ldots, \tau_{k+1}$, respectively, so that $\tau_{i+1} \preceq_{m} \cdots \preceq_{m} \tau_{k+1}$. Similarly, we extend $\sigma_{i-1}, \ldots, \sigma_{0}$ to $\tau_{i-1}, \ldots, \tau_{0}$. 
Remark. Before I proved Lemma 5.5, J. Pawlikowski pointed out that in the case when $\sigma \preceq_{n} \tau$ iff $\forall i<n \sigma(i) \leq \tau(i), \sigma, \tau \in 2^{n}$, one can get a perfect closed set linearly ordered by $\preceq$ by the following simple argument. (Lemmas 5.4 and 5.5 applied to this $\preceq$ will be used in the proof of Theorem 6.1.) Identify $\omega$ with the rationls, $\mathbb{Q}$. For any $r \in \mathbb{R}$, let $\alpha_{r} \in 2^{\omega}$ be the characteristic function of $\{q \in \mathbb{Q}: q<r\}$. Then $\left\{\alpha_{r}: r \in \mathbb{R}\right\}$ is a Borel uncountable subset of $2^{\omega}$ linearly ordered by $\preceq$. Now, any perfect closed subset of $\left\{\alpha_{r}: r \in \mathbb{R}\right\}$ works.

In the next theorem, we prove the existence of complete semicontinuous functions which are in a sense minimal. This result will not be used in the sequel; we nevertheless find it interesting.

Now, let $\preceq_{n}=$ the lexicographic order for each $n \in \omega$. Put $T_{l}=T_{\left(\preceq_{n}\right)}$. In this case, $\preceq$ is the lexicographic order on $2^{\omega}$; it linearly orders $2^{\omega}$. Let $K$ be a perfect, compact, metric space linearly ordered by a closed linear order. Fix $\psi: 2^{\omega} \rightarrow K$ to be a nondecreasing surjection such that

$$
\exists D \subset 2^{\omega} D \text { dense and } \psi \mid D \text { 1-to-1. }
$$

Put $F_{K}=\psi \circ F_{\left(\preceq_{n}\right)}$. By Lemma 5.3, $F_{K}$ is $K$-lsc complete. We show that it is in a sense a minimal such function.

Theorem 5.6. Let $f: X \rightarrow K$ be $K$-lsc complete, $K$ compact perfect. Then there is an embedding $\phi: T_{l} \rightarrow X$ such that $F_{K}=f \circ \phi$.

Proof. Let $D \subset X$ be as in Theorem 5.1. Without loss of generality we can assume that $D=X$. Define $h: 2^{<\omega} \rightarrow K$ by $h(\sigma)=\psi(\sigma * 00 \cdots)$. Let $S \subset \bigcup_{k} \prod_{n \leq k} 2^{n}$ be the pruned tree with $T_{l}=[S]$. For $\tau \in S$ we recursively, with respect to $l \bar{h}(\tau)$, define $U_{\tau} \subset X$ open and such that:

(i) $\operatorname{diam}\left(U_{\tau}\right) \leq 1 /(\operatorname{lh}(\tau)+1)$;

(ii) if $\tau_{1} \subset \tau_{2}$ and $\tau_{1} \neq \tau_{2}$, then $\bar{U}_{\tau_{1}} \subset U_{\tau_{2}}$, and if $\tau_{1}$ and $\tau_{2}$ are incompatible, then $U_{\tau_{1}} \cap U_{\tau_{2}}=\emptyset$;

(iii) $h(\tau(n-1)) \in f\left[U_{\tau}\right] \subset(h(\tau(n-1))-1 /(n+1), \infty)$.

Define $U_{\emptyset}$ to be any open set of diameter $<1$ containing an $x \in X$ such that $f(x)=\min K$. This is possible since $f$ is onto. If $U_{\tau}$ is defined, consider the set

$$
A=\left\{\tau^{\prime} \in S: \operatorname{lh}\left(\tau^{\prime}\right)=\operatorname{lh}(\tau)+1, \tau \subset \tau^{\prime}\right\} .
$$

Enumerate $A$ so that $A=\left\{\tau_{0}, \tau_{1}, \ldots, \tau_{m}\right\}$ for some $m \in \omega$, the $\tau_{i}$ 's are pairwise different, and $\tau_{0}(n) \preceq_{n} \tau_{1}(n) \preceq_{n} \cdots \preceq_{n} \tau_{m}(n)$, where $n=\operatorname{lh}(\tau)$. Note that by (5)

$$
h(\tau(n-1))=h\left(\tau_{0}(n)\right)<h\left(\tau_{1}(n)\right)<\cdots<h\left(\tau_{m}(n)\right) .
$$

Now, we find recursively $U_{\tau_{i}}, i \leq m$. Let $U_{\tau_{0}}^{\prime}$ and $V_{0}$ be open and such that ${\overline{U^{\prime}}}_{\tau_{0}} \cap \bar{V}_{0}=\emptyset,{\overline{U^{\prime}}}_{\tau_{0}}, \bar{V}_{0} \subset U_{\tau}, h\left(\tau_{0}(n)\right) \in h\left[U_{\tau_{0}}^{\prime}\right], h\left(\tau_{1}(n)\right) \in h\left[V_{0}\right]$, and $\operatorname{diam}\left(U_{\tau_{0}}^{\prime}\right)<$ $1 /(n+2)$. Put

$$
U_{\tau_{0}}=U_{\tau_{0}}^{\prime} \cap f^{-1}\left(\left(h\left(\tau_{0}(n)\right)-1 /(n+2), \infty\right)\right) .
$$

Then find $U_{\tau_{1}}^{\prime}$ and $V_{1}$ open and such that ${\overline{U^{\prime}}}_{\tau_{1}} \cap \bar{V}_{1}=\emptyset,{\overline{U^{\prime}}}_{\tau_{1}}, \bar{V}_{1} \subset V_{0}, h\left(\tau_{1}(n)\right) \in$ $h\left[U_{\tau_{1}}^{\prime}\right], h\left(\tau_{2}(n)\right) \in h\left[V_{1}\right]$, and $\operatorname{diam}\left(U_{\tau_{1}}^{\prime}\right)<1 /(n+2)$. Put

$$
U_{\tau_{1}}=U_{\tau_{1}}^{\prime} \cap f^{-1}\left(\left(h\left(\tau_{1}(n)\right)-1 /(n+2), \infty\right)\right) .
$$

Repeat this procedure $m+1$ times. 
Define $\phi$ by

$$
\phi(x)=\text { the unique element of } \bigcap_{n} U_{x \mid n} .
$$

By (i) and (ii), $\phi$ is continuous, and, by (ii), it is 1-to-1, whence it is an embedding since $T_{l}$ is compact. Since $f$ and $F_{K}$ are lsc, (iii) implies that $F_{K}=f \circ \phi$.

\section{The VAlue of dec For Baire Class 1 functions}

In [CMPS] it was proved that $\operatorname{dec}(P) \geq \operatorname{cov}(\mathcal{M})$, and in [St] that it is consistent that $\operatorname{dec}(P)>\operatorname{cov}(\mathcal{M})$. Thus $P$ provides a particularly simple example of a complicated Baire class 1 function. Below we show that $\operatorname{dec}(P)$ is actually highest possible. This answers two questions of Steprāns [St, Questions 7.1 and 7.2].

Theorem 6.1. $\operatorname{dec}(P)=\mathbf{d e c}$, where $P$ is Pawlikowski's function.

Proof. If $Y$ is a metric separable space, define

$$
\operatorname{dec}_{1 / 2}(Y)=\sup \{\operatorname{dec}(f): f: Y \rightarrow[0,1], f \text { lsc }\} .
$$

Of course, the value of $\operatorname{dec}_{1 / 2}(Y)$ would remain the same if we used usc instead of lsc functions in its definition.

First we show that $\mathbf{d e c}=\operatorname{dec}_{1 / 2}\left(2^{\omega}\right)$. The inequality $\geq$ is clear since each lsc is Baire class 1. To see $\leq$, first we show that $\operatorname{dec}_{1 / 2}(Y) \leq \operatorname{dec}_{1 / 2}\left(2^{\omega}\right)$ for any metric separable space $Y$. By a result due to Smirnov (see [E, Problem 1.8.G.]), $Y=\bigcup_{\alpha<\omega_{1}} Y_{\alpha}$, where $Y_{\alpha}, \alpha<\omega_{1}$, are zero-dimensional. Each $Y_{\alpha}$ embeds in $2^{\omega}$, and each lsc function on $Y_{\alpha}$ extends to $2^{\omega}$; thus, $\operatorname{dec}_{1 / 2}\left(Y_{\alpha}\right) \leq \operatorname{dec}_{1 / 2}\left(2^{\omega}\right)$. By a result of Adyan and Novikov, $\operatorname{dec}_{1 / 2}\left(2^{\omega}\right) \geq \aleph_{1}$ (see [JM, Theorem 4]); thus, we get

$$
\operatorname{dec}_{1 / 2}(Y) \leq \aleph_{1} \sup _{\alpha \in \omega_{1}} \operatorname{dec}_{1 / 2}\left(Y_{\alpha}\right) \leq \operatorname{dec}_{1 / 2}\left(2^{\omega}\right) \text {. }
$$

Now, let $f: \quad X \rightarrow Y$ be Baire class 1. Again by Smirnov's theorem, $Y=$ $\bigcup_{\alpha<\omega_{1}} Y_{\alpha}$ and each $Y_{\alpha}$ is zero-dimensional. Since $Y_{\alpha}$ embeds in [0,1], we can assume that $f \mid f^{-1}\left(Y_{\alpha}\right): f^{-1}\left(Y_{\alpha}\right) \rightarrow[0,1]$. By Lindenbaum's theorem (see [CMPS, Theorem 4.4]), any Baire class 1 function $h: Z \rightarrow[0,1]$ can be represented as $h=g_{2} \circ g_{1}$ where $g_{1}: Z \rightarrow[0,1]$ is usc and $g_{2}:[0,1] \rightarrow[0,1]$ is lsc, so $\operatorname{dec}(h) \leq \operatorname{dec}\left(g_{2}\right) \operatorname{dec}\left(g_{1}\right)$; whence

$$
\operatorname{dec}(f) \leq \aleph_{1} \sup _{\alpha<\omega_{1}} \operatorname{dec}_{1 / 2}\left(Y_{\alpha}\right) \operatorname{dec}_{1 / 2}([0,1]) \leq \operatorname{dec}_{1 / 2}\left(2^{\omega}\right)
$$

Thus dec $\leq \operatorname{dec}_{1 / 2}\left(2^{\omega}\right)$.

The theorem will be proved if we can show that $\operatorname{dec}_{1 / 2}\left(2^{\omega}\right) \leq \operatorname{dec}(P)$. Let $G: \omega^{\omega} \rightarrow 2^{\omega}$ be defined by

$$
G(x)(n)=\min \{1, x(n)\} \text { for } n \in \omega .
$$

Let $\preceq_{n}$ be the partial order on $2^{n}$ defined by

$$
\sigma \preceq_{n} \tau \text { iff } \forall i<n \sigma(i) \leq \tau(i) .
$$

Let $\preceq$ be the partial order on $2^{\omega}$ arising from $\left(\preceq_{n}\right)$ by formula $(3)$. Since $\left(\preceq_{n}\right)$ fulfils the assumptions of Lemma 5.5, there is a perfect, closed subset of $2^{\omega}$ linearly ordered by $\preceq$. Now, it follows from Lemma 5.4 that $\operatorname{dec}\left(F_{\left(\preceq_{n}\right)}\right) \geq \operatorname{dec}_{1 / 2}\left(2^{\omega}\right)$. Thus, it is enough to show that there is a homeomorphism $\phi:(\omega+1)^{\omega} \rightarrow 2^{\omega}$ such that $G \circ P=F_{\left(\preceq_{n}\right)} \circ \phi$ since then

$$
\operatorname{dec}(P) \geq \operatorname{dec}(G \circ P)=\operatorname{dec}\left(F_{\left(\preceq_{n}\right)}\right) \geq \operatorname{dec}_{1 / 2}\left(2^{\omega}\right) .
$$


Let $\eta \in(\omega+1)^{\omega}$. Put $\phi(\eta)=x$, where $x=(x(n)) \in \prod_{n} 2^{n}$, and for $i<n \in \omega$ we have

$$
x(n)(i)= \begin{cases}0, & \text { if } \eta(i) \geq n ; \\ 1, & \text { if } \eta(i)<n .\end{cases}
$$

It is easy to check that $\phi$ is continuous, 1 -to- 1 , and onto, whence, since $(\omega+1)^{\omega}$ is compact, $\phi$ is a homeomorphism. Now, $G \circ P(\eta)(i)=0$ iff $\eta(i)=\omega$ iff $\forall n>$ $i x(n)(i)=0$ iff $F_{\left(\preceq_{n}\right)}(x)(i)=0$.

Remarks. 1. Note that the second part of the above proof can be easily modified to show that there is a continuous function $G^{\prime}: \omega^{\omega} \rightarrow[0,1]$ such that $G^{\prime} \circ P$ : $(\omega+1)^{\omega} \rightarrow[0,1]$ is lsc complete.

2. It follows from Theorem 6.1, via the work of Steprāns [St, Definition 4.1, Proposition 4.1], that $\operatorname{dec}=\operatorname{cov}\left(\mathcal{J}_{p}\right)$, where $\mathcal{J}_{p}$ is a $\sigma$-ideal on $\omega^{\omega}$. The interesting fact about $\mathcal{J}_{p}$ is that its definition is purely combinatorial.

The following corollary is analogous to Corollary 3.7.

Corollary 6.2. Let $f: X \rightarrow Y$ be Baire class $1, X$ Souslin. Then $\operatorname{dec}(f) \leq \omega$ or $\operatorname{dec}(f)=$ dec.

Proof. If (i) of Theorem 4.1 holds, then $\operatorname{dec}(f) \leq \omega$. If (ii) holds, then $\operatorname{dec}(f)=\operatorname{dec}$ by Theorem 6.1 .

It was proved in [CMPS, Theorem 5.5] that there exists an lsc function $f$ such that $\operatorname{dec}(f) \geq \operatorname{cov}(\mathcal{M})$. We strengthen this result below. A function $f: Y \rightarrow[0,1]$, $Y$ a metric space, is called closed-to- 1 if $f^{-1}(y)$ is closed in $Y$ for any $y \in[0,1]$. Obviously, each continuous function is closed-to-1; however there exist plenty of closed-to-1, lsc functions which are not continuous, e.g., Pawlikowski's function $P$ being 1-to-1 is closed-to-1. The method of proof presented here is different from the one in [CMPS].

Theorem 6.3. Let $F: X \rightarrow[0,1]$ be lsc complete. If $\mathcal{F}$ is a family of subsets of $X$ such that $\bigcup \mathcal{F}=X$, and $F \mid Y$ is closed-to- 1 for any $Y \in \mathcal{F}$, then $|\mathcal{F}| \geq \operatorname{cov}(\mathcal{M})$.

Proof. Let $\left\{V_{n}: n \in \omega\right\}$ be a countable topological basis of $X$. Let $D$ be as in Theorem 5.1. Without loss of generality we can assume that $D=X$. Fix $n \in \omega$ and $Y \in \mathcal{F}$. We claim that there is at most one $y \in[0,1]$ such that $V_{n} \cap F^{-1}(y) \neq \emptyset$ and $Y$ is dense in $V_{n} \cap F^{-1}(y)$. If not, let $y_{1}<y_{2}$ be two such $y$ 's. Since (1) from the proof of Theorem 5.1 holds, we have

$$
Y \cap V_{n} \cap F^{-1}\left(y_{1}\right) \subset \overline{Y \cap V_{n} \cap F^{-1}\left(y_{2}\right)},
$$

whence, since $Y \in \mathcal{F}, F \mid\left(Y \cap V_{n} \cap F^{-1}\left(y_{1}\right)\right) \equiv y_{2}$, a contradiction. Thus, we can pick $y_{0} \in[0,1]$ such that for any $n \in \omega$ and any $Y \in \mathcal{F}$ either $V_{n} \cap F^{-1}\left(y_{0}\right)=\emptyset$, or $Y$ is not dense in $V_{n} \cap F^{-1}\left(y_{0}\right)$. Then, clearly, $Y$ is nowhere dense in $F^{-1}\left(y_{0}\right)$. Since $F^{-1}\left(y_{0}\right)$ is $\Pi_{2}^{0}$, as $F$ is Baire class 1 , and $\bigcup \mathcal{F} \supset F^{-1}\left(y_{0}\right)$, we have $|\mathcal{F}| \geq \operatorname{cov}(\mathcal{M})$.

Below, we prove a result which relates the value of dec to the value of an ordinal rank on the family of all Baire class 1 functions. The ordinal rank, called the oscillation rank and denoted by $\beta$, was studied in great detail in $[\mathrm{KL}]$. First, let us recall its definition. Let $f: X \rightarrow \mathbb{R}$. For $P \subset X$ and $\epsilon>0$, define $P_{\epsilon, f}^{*}=\{x \in P: \operatorname{osc}(f \mid P, x) \geq \epsilon\}$. (By $\operatorname{osc}(g, x)$ we denote the oscillation of 
the function $g$ at $x$.) Now by iterating this operation, we obtain a sequence $P_{\epsilon, f}^{\alpha}$, $\alpha<\omega_{1}$ :

$$
P_{\epsilon, f}^{\alpha+1}=\left(P_{\epsilon, f}^{\alpha}\right)^{*} \text { and } P_{\epsilon, f}^{\lambda}=\bigcap_{\alpha<\lambda} P_{\epsilon, f}^{\alpha} \text { if } \lambda \text { is limit. }
$$

Now let $\beta(f, \epsilon)=$ least $\alpha$ with $X_{\epsilon, f}^{\alpha}=\emptyset$ if such an $\alpha$ exists, and $\beta(f, \epsilon)=\omega_{1}$ if there is no such $\alpha$. Put $\beta(f)=\sup _{\epsilon>0} \beta(f, \epsilon)$. Recall [KL, Section 1, Proposition 2 ] that $f$ is Baire class 1 iff $\beta(f)<\omega_{1}$. Now, we state the result.

Proposition 6.4. (i) Let $f: X \rightarrow \mathbb{R}, X$ Polish, be Baire class 1. Assume $\beta(f)<$ $\omega$. Then $\operatorname{dec}(f) \leq \omega$.

(ii) $\beta(P)=\omega$ (when $P$ is considered as a real function by appropriately embedding its range into $\mathbb{R})$.

Proof. To see (i), put $\beta(f)=n$. Then there exists $\epsilon_{0}>0$ such that $\beta(f, \epsilon)=n$ for $\epsilon<\epsilon_{0}$. Let $X_{k}^{1}=\{x \in X: \operatorname{osc}(f, x)<1 / k\}$ and

$$
X_{k}^{m}=\left\{x \in X \backslash \bigcup_{i=1}^{m-1} X_{k}^{i}: \operatorname{osc}\left(f \mid X \backslash \bigcup_{i=1}^{m-1} X_{k}^{i}, x\right)<1 / k\right\}
$$

for $m \leq n$ and for $k$ with $1 / k<\epsilon_{0}$. By our assumption, $\bigcup_{i=0}^{n} X_{k}^{i}=X$ for any $k$. Now define $A_{1}=\bigcap_{k=1}^{\infty} X_{k}^{1}$ and

$$
A_{l}^{m}=\left(\bigcap_{j=1}^{\infty} \bigcup_{i=1}^{m} X_{j}^{i}\right) \backslash\left(\bigcap_{j=1}^{l} \bigcup_{i=1}^{m-1} X_{j}^{i}\right)
$$

for $1<m \leq n$ and $l<\omega$. Notice that $A_{1} \cup \bigcup_{m=2}^{n} \bigcup_{l=1}^{\infty} A_{l}^{m}=X$; thus, it is enough to see that $f \mid A_{1}$ and $f \mid A_{l}^{m}, l<\omega, 1<m \leq n$, are continuous. But we have $A_{1} \subset X_{k}^{1}$ for all $k<\omega$. Also, if $k>j$, then $\bigcup_{i=1}^{m-1} X_{k}^{i} \subset \bigcup_{i=1}^{m-1} X_{j}^{i}$ for $m \leq n$. Thus, for all $k>l$,

$$
A_{l}^{m} \subset \bigcup_{i=1}^{m} X_{k}^{i} \backslash \bigcup_{i=1}^{m-1} X_{k}^{i}=X_{k}^{m}
$$

Therefore, $\operatorname{osc}\left(f \mid A_{1}, x\right)=0$ for $x \in A_{1}$ and $\operatorname{osc}\left(f \mid A_{l}^{m}, x\right)=0$ for $x \in A_{l}^{m}$.

Now we show (ii). It is not difficult to find an embedding $\phi: \omega^{\omega} \rightarrow \mathbb{R}$ such that for any $\sigma \in \omega^{n}, n \in \omega, \operatorname{diam}\left(\phi\left[\left\{\eta \in \omega^{\omega}: \sigma \subset \eta\right\}\right]\right)<2^{-n}$. Consider $\phi \circ P:(\omega+1)^{\omega} \rightarrow \mathbb{R}$. For $k \leq n \in \omega$ define

$$
A_{k}=\left\{\eta \in(\omega+1)^{\omega}:|\{i<n: \eta(i)=\omega\}| \geq k\right\} .
$$

Note that $A_{n}=\emptyset$. By directly calculating the result of applying ( )* to $(\omega+1)^{\omega} k$ times, $k \leq n$, with $\epsilon=2^{-n}$ and $f=\phi \circ P$, we obtain that $\left((\omega+1)^{\omega}\right)_{2^{-n}, \phi \circ P}^{k} \subset A_{k}$. Thus, $\beta\left(\phi \circ P, 2^{-n}\right) \leq n$. It follows that $\beta(\phi \circ P) \leq \omega$. Since $\operatorname{dec}(\phi \circ P)=\operatorname{dec}(P)>\omega$, we get from (i) that $\beta(\phi \circ P) \geq \omega$ as well.

\section{Applications to measures}

Let $\lambda$ be the Lebesgue measure on $[0,1]$. Then the restriction of $\lambda$ to $K([0,1])$ is usc. We denote this restriction by the same letter $\lambda$. Van Mill and Pol proved in [vMP, Theorem 3.1] that $\lambda$ is usc complete. (Actually, they showed that for any compact, metric space $X$, not only $2^{\omega}$, and any usc function $f: X \rightarrow[0,1]$ there is a continuous function $\phi: X \rightarrow K([0,1])$ such that $f=\lambda \circ \phi$.) Below we are able to generalize this result using the characterization from Theorem 5.1. Let $X$ 
be a compact, metric space. Recall that a function $c: K(X) \rightarrow[0,1]$ is called a capacity if

(i) $c\left(F_{1}\right) \leq c\left(F_{2}\right)$ for $F_{1}, F_{2} \in K(X)$ with $F_{1} \subset F_{2}$;

(ii) $c\left(\bigcap_{n} F_{n}\right)=\inf _{n} c\left(F_{n}\right)$ for any sequence $F_{n} \in K(X), n \in \omega$, with $F_{n+1} \subset F_{n}$;

(iii) if $F \in K(X)$ and $F=\bigcup_{n} F_{n}$ for some sequence $F_{n} \in K(X), n \in \omega$, with $F_{n} \subset F_{n+1}$, then $c(F)=\sup _{n} c\left(F_{n}\right)$.

Notice that the restriction of any probability, Borel measure on $X$ to $K(X)$ is a capacity; however, there exist lots of important capacities which cannot be obtained in this way.

Corollary 7.1. Let $X$ be a compact, metric space. Let $c: K(X) \rightarrow[0,1]$ be a capacity. Assume that $c(X)=1$ and $c(D)=0$ for any finite set $D \subset X$. Then $c$ is usc complete.

Proof. First notice that conditions (i) and (ii) guarantee that $c$ is usc. Thus, by Theorem 5.1 and the remark following its proof, it is enough to check that for any open set $U \subset K(X), c[U]$ is of the form $\left[0, r_{0}\right)$ or $\left[0, r_{0}\right]$ for some $r_{0} \in[0,1]$. Let $F_{0} \in U$. We will show that for any real $r$ with $c\left(F_{0}\right) \geq r \geq 0$ there is $F^{\prime} \in U$ with $c\left(F^{\prime}\right)=r$. We can easily find $D \subset F_{0}$ finite such that for any $F \in K(X)$ if $D \subset F \subset F_{0}$, then $F \in U$. Let $\mathcal{F}$ be a maximal, linearly ordered by inclusion family of closed subsets $F$ of $X$ such that $D \subset F \subset F_{0}$ and $c(F) \geq r$. Put $F^{\prime}=\bigcap \mathcal{F}$. Then $F^{\prime} \in U$. We can find a decreasing sequence $F_{n} \in \mathcal{F}, n \in \omega$, such that $F^{\prime}=\bigcap_{n} F_{n}$; thus, by (ii), $c\left(F^{\prime}\right) \geq r$. If $F^{\prime}$ is finite, then $r=0$ and $c\left(F^{\prime}\right)=r$. Otherwise, we can find a decreasing sequence of open sets $V_{n}, n \in \omega$, such that $D \cap V_{n}=\emptyset$, $F^{\prime} \cap V_{n} \neq \emptyset$, and $\bigcap_{n} V_{n}=\emptyset$. Put $F_{n}=F^{\prime} \backslash V_{n}$. Then by the definition of $F^{\prime}$, $c\left(F_{n}\right)<r$. By (iii), $c\left(F^{\prime}\right)=\sup _{n} c\left(F_{n}\right) \leq r$. Thus $c\left(F^{\prime}\right)=r$.

Jackson and Mauldin proved in [JM, Theorem 5] that $\operatorname{dec}(\lambda)>\omega$, where $\lambda$ is the restriction to $K([0,1])$ of the Lebesgue measure on $[0,1]$. It follows from van Mill-Pol's result [vMP, Theorem 3.1] mentioned above that $\operatorname{dec}(\lambda)=$ dec. In the next theorem, using Corollary 7.1 and Theorem 6.1, we characterize those Borel, probability measures $\mu$ on compact, metric spaces for which $\operatorname{dec}(\mu)=$ dec. By $\delta_{x}$ we denote the Dirac measure concentrated at $x$, i.e., $\delta_{x}(A)=1$ if $x \in A$ and $\delta_{x}(A)=0$ otherwise.

Theorem 7.2. Let $\mu$ be a Borel, probability measure on a compact metric space $X$. Let the same letter denote the restriction of $\mu$ to $K(X)$. Then $\operatorname{dec}(\mu)=\mathbf{d e c}$ unless $\mu=\sum_{x \in D} \alpha_{x} \delta_{x}$ where $\alpha_{x}>0, \sum_{x \in D} \alpha_{x}=1$, and $\{x \in D: x$ is not isolated $\}$ is finite. If $\mu$ is of this form, then $\operatorname{dec}(\mu)=n+1$ where $n=\mid\{x \in D$ : $x$ is not isolated $\}$.

Proof. If $\mu$ is not purely atomic, then there is a closed set $F_{0} \subset X$ such that $\mu\left(F_{0}\right)>0$ and $\mu(\{x\})=0$ for any $x \in F_{0}$. Then by Corollary 7.1 ,

$$
\frac{1}{\mu\left(F_{0}\right)} \mu \mid\left\{F \in K(X): F \subset F_{0}\right\}
$$

is usc complete. It follows that $\operatorname{dec}(\mu)=\mathbf{d e c}$.

Put $N=\{x \in X: x$ is not isolated and $\mu(\{x\}) \neq 0\}$. Assume $N$ is infinite. We will find a continuous function $\phi:(\omega+1)^{\omega} \rightarrow K(X)$ such that if $\eta_{k}, \eta \in(\omega+1)^{\omega}$, $\eta_{k} \rightarrow \eta$, then $P\left(\eta_{k}\right) \nrightarrow P(\eta)$ implies $\mu \circ \phi\left(\eta_{k}\right) \nrightarrow \mu \circ \phi(\eta)$. Then, clearly, if $\mu \mid Y$ is continuous, so is $P \mid \phi^{-1}(Y)$; thus, $\operatorname{dec}(\mu) \geq \operatorname{dec}(P)$, and we are done by Theorem 
6.1. Find a converging sequence $x_{\omega}^{n} \in N, n \in \omega$. Put $y=\lim _{n} x_{\omega}^{n}$. Find $x_{k}^{n}, k \in \omega$, with $x_{k}^{n} \rightarrow x_{\omega}^{n}$. By choosing subsequences, we can assume that

(i) $\forall k, l \in \omega+1 \forall n, m \in \omega x_{k}^{n} \neq x_{l}^{m}$ if $k \neq l$ or $n \neq m$;

(ii) $\forall k \in \omega+1 \forall n \in \omega d\left(x_{k}^{n}, y\right) \leq 1 / n$;

(iii) $\forall n \in \omega \mu\left(\left\{x_{k}^{m}: n<m \in \omega, k \in \omega+1\right\}\right)<\mu\left(\left\{x_{\omega}^{n}\right\}\right)$.

Define $\phi:(\omega+1)^{\omega} \rightarrow K(X)$ by

$$
\phi(\eta)=\{y\} \cup\left\{x_{\eta(n)}^{n}: n \in \omega\right\} \text { for } \eta \in(\omega+1)^{\omega} .
$$

By (ii), the set on the right hand side is closed. It is routine to check that $\phi$ is continuous. Let $\eta_{k}, \eta \in(\omega+1)^{\omega}, \eta_{k} \rightarrow \eta$. Assume $P\left(\eta_{k}\right) \nrightarrow P(\eta)$. Then there is an $n \in \omega$ such that $\eta_{k}(n) \in \omega$ for infinitely many $k, \eta_{k}(n) \rightarrow \omega$, and $\eta(n)=\omega$. Let $n_{0}$ be the smallest such $n$. Without loss of generality we can assume that $\eta_{k}(n)=\eta(n)$ for all $n<n_{0}$ and $\eta_{k}\left(n_{0}\right) \in \omega$ for all $k \in \omega$. Then

$$
\begin{aligned}
\mu \circ \phi\left(\eta_{k}\right) \leq & \mu\left(\left\{x_{\eta(n)}^{n}: n<n_{0}\right\}\right)+\mu\left(\left\{x_{\eta_{k}\left(n_{0}\right)}^{n_{0}}\right\}\right) \\
& +\mu\left(\left\{x_{l}^{n}: l \in \omega+1, n \in \omega, n>n_{0}\right\}\right)+\mu(\{y\}) .
\end{aligned}
$$

Since $\eta_{k}\left(n_{0}\right) \rightarrow \omega$, by (i), $\mu\left(\left\{x_{\eta_{k}\left(n_{0}\right)}^{n_{0}}\right\}\right) \rightarrow 0$; thus,

$$
\begin{aligned}
\limsup _{k} \mu \circ \phi\left(\eta_{k}\right) \leq & \mu\left(\left\{x_{\eta(n)}^{n}: n<n_{0}\right\}\right) \\
& +\mu\left(\left\{x_{l}^{n}: l \in \omega+1, n \in \omega, n>n_{0}\right\}\right)+\mu(\{y\}) .
\end{aligned}
$$

On the other hand, by (iii),

$$
\begin{aligned}
\mu \circ \phi(\eta) & =\mu\left(\left\{x_{\eta(n)}^{n}: n<n_{0}\right\}\right)+\mu\left(\left\{x_{\omega}^{n_{0}}\right\}\right)+\mu(\{y\}) \\
& >\mu\left(\left\{x_{\eta(n)}^{n}: n<n_{0}\right\}\right)+\mu\left(\left\{x_{l}^{n}: l \in \omega+1, n \in \omega, n>n_{0}\right\}\right)+\mu(\{y\}) .
\end{aligned}
$$

Therefore, $\mu \circ \phi\left(\eta_{k}\right) \not \mu \circ \phi(\eta)$.

If $|N|=n<\aleph_{0}$, put

$$
X_{i}=\{F \in K(X):|F \cap N|=i\}, \text { for } i \in\{0, \ldots, n\} .
$$

It is easy to check that $\mu \mid X_{i}$ is continuous, so $\operatorname{dec}(\mu) \leq n+1$. To show that $\operatorname{dec}(\mu) \geq n+1$, assume towards a contradiction that $\operatorname{dec}(\mu) \leq n$. Let $Y_{0}, \ldots, Y_{n-1}$ be such that $\mu \mid Y_{i}$ is continuous and $\bigcup_{i=0}^{n-1} Y_{i}=K(X)$. Now find an open set $U \supset N$ such that

$$
\mu(U \backslash N)<\min \{\mu(\{x\}): x \in N\} .
$$

Notice that

- for any $A \subset N$ the set $\{F \in K(X): F \subset U, F \cap N=A\}$ is $\Pi_{2}^{0}$, so the Baire Category Theorem is true for it;

- if $A \subset A^{\prime} \subset N$, then

$$
\left\{F \in K(X): F \subset U, F \cap N=A^{\prime}\right\} \subset \overline{\{F \in K(X): F \subset U, F \cap N=A\}} .
$$

(This holds since the points in $N$ are not isolated.)

Using this, we recursively construct $A_{j} \subset N$ and $Z_{j} \subset\{F \in K(X): F \subset$ $\left.U, F \cap N=A_{j}\right\}, j \in\{0, \ldots, n\}$, so that

(iv) $\left|A_{j}\right|=j$;

(v) $A_{j} \subset A_{j+1}$ for $j<n$;

(vi) $\forall j \leq n \exists i \leq n-1 Z_{j} \subset Y_{i}$; 
(vii) $\bar{Z}_{j}$ contains a nonempty, relatively open subset of $\{F \in K(X): F \subset U, F \cap$ $\left.N=A_{j}\right\}$

(viii) $Z_{j+1} \subset \bar{Z}_{j}$ for $j<n$.

Using (vi), by the pigeonhole principle, we get $j_{1}<j_{2} \leq n$ and $i_{0} \leq n-1$ with $Z_{j_{1}}, Z_{j_{2}} \subset Y_{i_{0}}$. Let $x_{0} \in A_{j_{2}} \backslash A_{j_{1}}$. Then

$$
\mu \mid Z_{j_{2}} \geq \mu\left(A_{j_{2}}\right) \geq \mu\left(A_{j_{1}}\right)+\mu\left(\left\{x_{0}\right\}\right) .
$$

On the other hand,

$$
\mu \mid Z_{j_{1}} \leq \mu\left(A_{j_{1}}\right)+\mu(U \backslash N)
$$

whence, since $\mu \mid Y_{i_{0}}$ is continuous,

$$
\mu \mid\left(Y_{i_{0}} \cap \bar{Z}_{j_{1}}\right) \leq \mu\left(A_{j_{1}}\right)+\mu(U \backslash N) .
$$

But (7) and (8) contradict (6), since, by (viii), $Z_{j_{2}} \subset Y_{i_{0}} \cap \bar{Z}_{j_{1}}$.

\section{REFERENCES}

[AN] S.I. Adyan and P.S. Novikov, On a semicontinuous function, Zap. MPGI W.I. Lenina 138 (1958), 3-10. MR 22:11081

[BD] H. Becker and R. Dougherty, On disjoint Borel uniformizations, Adv. Math. (to appear).

[CM] J. Cichoń and M. Morayne, Universal functions and generalized classes of functions, Proc. Amer. Math. Soc. 102 (1988), 83-89. MR 89c:26003

[CMPS] J. Cichon, M. Morayne, J. Pawlikowski, and S. Solecki, Decomposing Baire functions, J. Symb. Logic 56 (1991), 1273-1283. MR 92j:04001

[E] R. Engelking, Dimension Theory, North-Holland, 1978. MR 58:2753b

[HKL] L. Harrington, A.S. Kechris, and A. Louveau, A Glimm-Effros dichotomy for Borel equivalence relations, J. Amer. Math. Soc. 3 (1990), 903-927. MR 91h:28023

[HOR] R. Haydon, E. Odell, and H. P. Rosenthal, Certain subclasses of Baire-1 functions with Banach space applications, Lecture Notes in Mathematics 1470, Springer-Verlag, 1990, pp. 1-35. MR 92h:46018

[JM] S. Jackson and R.D. Mauldin, Some complexity results in topology and analysis, Fund. Math. 141 (1992), 75-83. MR 93i:03067

[JR] J.E. Jayne and C.A. Rogers, First level Borel functions and isomorphisms, J. Math. pures et appl. 61 (1982), 177-205. MR 84a:54072

[K] L. Keldiš, Sur les fonctions premières measurables B, Dokl. Akad. Nauk. SSSR 4 (1934), 192-197.

[KL] A.S. Kechris and A. Louveau, A classification of Baire class 1 functions, Trans. Amer. Math. Soc. 318 (1990), 209-236. MR 90f:26005

[L1] A. Louveau, A separation theorem for $\Sigma_{1}^{1}$ sets, Trans. Amer. Math. Soc. 260 (1980), 363-378. MR 81j:04001

[L2] A. Louveau, Some results in the Wadge hierachy of Borel sets, Cabal Seminar 79-81, Lecture Notes in Mathematics 1019, Springer-Verlag, 1983, pp. 28-55. MR 86j:03002

[MK] D.A. Martin and A.S. Kechris, Infinite games and effective descriptive set theory, Analytic Sets, Academic Press, London, 1980, pp. 403-470. MR 82m:03063

[vMP] J. van Mill and R. Pol, Baire 1 functions which are not countable unions of continuous functions, Acta Math. Hungar. 66 (1995), 289-300. MR 96a:54017

[M] M. Morayne, Algebras of Borel measurable functions, Fund. Math. 141 (1992), 229-242. MR 93j:26003

[R] H.P. Rosenthal, Some recent discoveries in the isomorphic theory of Banach spaces, Bull. Amer. Math. Soc. 84 (1978), 803-831. MR 80d:46023

[R1] H.P. Rosenthal, A characterization of Banach spaces containing $c_{0}$, J. Amer. Math. Soc. 7 (1994), 707-748. MR 94i: 46032

[R2] H.P. Rosenthal, Differences of bounded semi-continuous functions, I (to appear).

[S] S. Solecki, Covering analytic sets by families of closed sets, J. Symb. Logic 59 (1994), 1022-1031. MR 95g:54033 
[SS] S. Shelah and J. Steprāns, Decomposing Baire class 1 functions into continuous functions, Fund. Math. 145 (1994), 171-180. MR 95i:03110

[St] J. Steprāns, A very discontinuous Borel function, J. Symb. Logic 58 (1993), 1268-1283. MR 95c:03120

[Sr] J. Stern, Évaluation du rang de Borel de certains ensembles, C. R. Acad. Sc. Paris Série A 286 (1978), 855-857. MR 81e:03049

Department of Mathematics, Indiana University, Bloomington, Indiana 47405

E-mail address: ssolecki@indiana.edu 